OPEN ACCESS

Edited by:

Maria Manuela Rosado,

Sapienza University of Rome, Italy

Reviewed by:

Siegfried Weiss,

Helmholtz Association of German Research Centers (HZ), Germany Jessica Naomi Lancaster, Mayo Clinic Arizona, United States

${ }^{*}$ Correspondence:

Palmira Barreira-Silva palmirasilva@med.uminho.pt

Margarida Correia-Neves mcorreianeves@med.uminho.pt

${ }^{\dagger}$ Deceased

Specialty section:

This article was submitted to

T Cell Biology,

a section of the journal

Frontiers in Immunology

Received: 16 April 2021 Accepted: 30 November 2021 Published: 20 December 2021

Citation:

Barreira-Silva $P$, Melo-Miranda $R$, Nobrega $C$, Roque $S$,

Serre-Miranda $C$, Borges $M$,

Armada G, de Sá Calçada D,

Behar SM, Appelberg $R$ and Correia-Neves M (2021) IFN $\gamma$ and iNOS-Mediated Alterations in the Bone Marrow and Thymus and Its Impact on Mycobacterium avium-Induced Thymic Atrophy.

Front. Immunol. 12:696415. doi: 10.3389/fimmu.2021.696415

\section{IFN $\gamma$ and iNOS-Mediated Alterations in the Bone Marrow and Thymus and Its Impact on Mycobacterium avium-Induced Thymic Atrophy}

\author{
Palmira Barreira-Silva ${ }^{1,2^{*}}$, Rita Melo-Miranda ${ }^{1,2}$, Claudia Nobrega ${ }^{1,2}$, Susana Roque ${ }^{1,2}$, \\ Cláudia Serre-Miranda ${ }^{1,2}$, Margarida Borges ${ }^{3}$, Gisela Armada ${ }^{1,2}$, Daniela de Sá Calçada ${ }^{1,2}$, \\ Samuel M. Behar ${ }^{4}$, Rui Appelberg ${ }^{5,6,7 \dagger}$ and Margarida Correia-Neves ${ }^{1,2,8^{*}}$ \\ ${ }^{1}$ Life and Health Sciences Research Institute (ICVS), School of Medicine, University of Minho, Braga, Portugal, \\ 2 Life and Health Sciences Research Institute/Biomaterials, Biodegradables and Biomimetics Research Group (ICVS/3B's), \\ PT Government Associate Laboratory, Braga, Portugal, ${ }^{3}$ Research Unit on Applied Molecular Biosciences (UCIBIO)/Rede de \\ Química e Tecnologia (REQUINTE), Departamento de Ciências Biológicas, Faculdade de Farmácia, Universidade do Porto, \\ Porto, Portugal, ${ }^{4}$ Department of Microbiology and Physiological Systems, University of Massachusetts Medical School, \\ Worcester, MA, United States, 5 Instituto de Investigação e Inovação em Saúde (i3S), Instituto de Investigação e Inovação \\ em Saúde, Universidade do Porto, Porto, Portugal, ${ }^{6}$ IBMC-Instituto de Biologia Molecular e Celular, Universidade do Porto, \\ Porto, Portugal, 7 ICBAS-Instituto de Ciências Biomédicas Abel Salazar, Universidade do Porto, Porto, Portugal, ${ }^{8}$ Division of \\ Infectious Diseases, Department of Medicine Solna, Karolinska Institutet, Stockholm, Sweden
}

Disseminated infection with the high virulence strain of Mycobacterium avium 25291 leads to progressive thymic atrophy. We previously showed that $M$. avium-induced thymic atrophy results from increased glucocorticoid levels that synergize with nitric oxide (NO) produced by interferon gamma (IFNy) activated macrophages. Where and how these mediators act is not understood. We hypothesized that IFNy and NO promote thymic atrophy through their effects on bone marrow (BM) T cell precursors and T cell differentiation in the thymus. We show that $M$. avium infection cause a reduction in the percentage and number of common lymphoid progenitors (CLP). Additionally, BM precursors from infected mice show an overall impaired ability to reconstitute thymi of RAGKO mice, in part due to IFN $\gamma$. Thymi from infected mice present an IFN $\gamma$ and NO-driven inflammation. When transplanted under the kidney capsule of uninfected mice, thymi from infected mice are unable to sustain T cell differentiation. Finally, we observed increased thymocyte death via apoptosis after infection, independent of both IFN $\gamma$ and iNOS; and a decrease on active caspase-3 positive thymocytes, which is not observed in the absence of iNOS expression. Together our data suggests that $M$. avium-induced thymic atrophy results from a combination of defects mediated by IFN $\gamma$ and NO, including alterations in the BM T cell precursors, the thymic structure and the thymocyte differentiation.

Keywords: thymus premature atrophy, Mycobacterium avium infection, IFN gamma, nitric oxide, BM T cell precursors, thymocyte differentiation

\section{INTRODUCTION}

$\mathrm{T}$ cell progenitors migrate from the bone marrow (BM) to the thymus, where they encounter a specialized environment that promotes their differentiation into new $\mathrm{T}$ cells. The thymus undergoes physiological involution with aging $(1,2)$. This, together with reports from late 1990s showing that removal of the thymus at an early age seemed to have little consequence on the normal $\mathrm{T}$ cell 
repertoire of adults, led to the idea that the thymus was only needed for $\mathrm{T}$ cell production early in life (3). However, further investigation showed that thymectomy early in life leads to premature immune aging (4). Importantly, the amount of functional thymic tissue correlates with the reconstitution of the peripheral $\mathrm{T}$ cell repertoire after severe lymphopenia, as is the case of patients with acquired immunodeficiency syndrome (AIDS) under antiretroviral therapy, or in patients with cancer after BM transplant $(5,6)$. These data highlight the importance of understanding premature thymic atrophy, which can affect the overall immune health of the individual.

A variety of pathogens target the thymus and induce premature thymic atrophy in humans, such as the Human Immunodeficiency Virus (HIV), and experimental animal models, such as the bacterium Mycobacterium avium, the fungus Paracoccidioids brasiliensis, the parasite Tripanosoma cruzi, and the virus Murine Leukemia Virus (MuLV), among others $(7,8)$. Although the thymus is a target for infection with different mycobacteria (9), M. avium-induced thymic atrophy has been shown to be strain-dependent (10). For example, premature thymic atrophy develops after infection with the high virulence strain $M$. avium subspecies avium strain 25291, but no signs of atrophy develop even after several months of chronic infection with the low virulence strain $2447(10,11)$.

$M$. avium infection-induced thymic atrophy arises from a synergy between glucocorticoids (GC) and nitric oxide (NO) produced by interferon gamma (IFN $\gamma$ ) activated macrophages $(\mathrm{M} \phi)$, since mice lacking IFN $\gamma$, iNOS or the activation of M $\mathrm{M}$ by IFN $\gamma$ show no infection-induced thymic atrophy (10). However, where and how these mediators act to lead to M. avium infection-induced thymic atrophy is unknown.

Different alterations in the thymus occur during premature thymic atrophy observed in different mouse models of infection $(7,8,12)$. Mechanisms that have been defined include: (i) thymic structural changes $(13,14)$; (ii) reduction in thymocyte proliferation (15); (iii) increased thymocyte cell death (16-19); and, (iv) increased export of immature thymocytes to the periphery (20-22). A frequently described mechanism is GC overproduction that causes excessive death of thymocytes, mostly at the double positive (DP) stage $(23,24)$. Additionally, IFN $\gamma$ and NO can also induce thymocyte cell death, either independently or by synergizing with GC $(16,25-28)$.

A possible mechanism of premature thymic atrophy, which has been seldom explored, is alterations of $\mathrm{T}$ cell precursors in the BM, and could include: (i) decreased production; (ii) arrest in the $\mathrm{BM}$ and consequent decreased migration and/or (iii) loss of ability to populate the thymus. In fact, infection-induced alterations of the BM have been abundantly documented following infection by bacteria including Mycobacterium tuberculosis (29), M. avium (30), Group A Streptococcus (31) and Escheriachia coli (32); viruses including the HIV (33), Pneumovirus (34) and Vaccinia virus (35); and parasites such as Plasmodium chabaudi (36), Plasmodium berghei (37) and Trypanosoma brucei (38). IFN $\gamma$ is the major mediator produced during some of these infections that promotes alterations in BM cells $(30,31,34,36)$, via the expansion of LSK (Lineage ${ }^{-} \mathrm{Sca}^{+}$
$\mathrm{cKit}^{+}$) cells and the increased differentiation of cells from the myeloid lineage (39). Other molecules such as tumor necrosis factor (TNF), NO and type I IFN have also been associated with $\mathrm{BM}$ alterations during infection $(34,38,40)$. However, except for two reports showing that the reduction of $\mathrm{BM}$ precursors is associated with sepsis-induced and T. cruzi infection-induced thymic atrophy $(41,42)$, we are not aware of other reports that associate alterations in the BM with infection-induced thymic atrophy.

We previously showed that M. avium strain 25291 infected wild type (WT) mice have a reduction in the number of early thymic precursors (ETP) (10), which is the most immature cell population in the thymus. Additionally, mice that lack iNOS expression (iNOSKO mice) are resistant to infection-induced thymic atrophy, but have also reduced numbers of ETP (10). While these data indicate that alterations in the BM $\mathrm{T}$ cell precursors may not be sufficient to cause premature thymic atrophy, it could be an important step in the process. Here, we took advantage of $\mathrm{KO}$ mouse strains, cell transfer and thymic transplant models to study how alterations mediated by IFN $\gamma$ and/or iNOS affecting the thymic environment and/or the $\mathrm{BM}$ contribute to premature thymic atrophy during $M$. avium infection.

\section{MATERIALS AND METHODS}

\section{Mice and Infection}

C57BL/6 WT mice were purchased from Charles River Laboratories (Barcelona, Spain) or bred at the Life and Health Sciences Research Institute (ICVS - School of Medicine, University of Minho, Braga, Portugal) from a breeding pair purchased from Charles River Laboratories. B6.SJL-Ptprca Pepcb/BoyJ (WT CD45.1) and IFN $\gamma-\mathrm{KO}$ C57BL/6 mice were bred at ICVS from a breeding pair purchased from The Jackson Laboratory (Bar Harbor, ME, USA). Transgenic mice with a selective impairment on IFN $\gamma$ signaling in $\mathrm{CD}^{+} 8^{+}$cells (MIIG) (43) were bred at the Institute for Molecular and Cell Biology (University of Porto, Porto, Portugal) from a breeding pair provided by the Cincinnati Children's Hospital Medical Center and the University of Cincinnati College of Medicine (Cincinnati, OH, USA). iNOS-deficient C57BL/6 (iNOSKO) (44) mice were bred at ICVS after back-crossing the original strain (kindly provided by Drs. J. Mudgett, J.D. MacMicking, and C. Nathan, Cornell University, New York, NY, USA) onto a C57BL/ 6 background for seven generations, or purchased from The Jackson Laboratory. B6(Cg)-Rag $2^{\text {tm1.1Cgn }}(\mathrm{RAGKO})$ mice were bred at ICVS from a breeding pair purchased from Instituto Gulbenkien da Ciência (Oeiras, Portugal) or from The Jackson Laboratory. iNOS and Rag2-double deficient (iNOS.RAG.2KO) mice were obtained, at ICVS, after crossing F1 resulting from the cross of iNOSKO and RAGKO mice.

Eight- to ten-week-old female mice were infected intravenously (i.v.) through a lateral tail vein with $10^{6}$ colony forming units (CFU) of the M. avium strain ATCC $25291 \mathrm{SmT}$ (obtained from the American Type Culture Collection, 
Manassas, VA, USA) or the strain 2447 (provided by Dr. F. Portaels, Institute of Tropical Medicine, Antwerp, Belgium). Bacterial inocula preparation and bacterial load quantification in the organs was performed as previously described $(11,45)$. No signs of major distress were observed for the first 2 months upon infection, though some animals showed signs of deterioration of body condition in a non-synchronous way. To avoid excessive and unnecessary suffering of animals, humane endpoints were applied, and mice were euthanized when reaching 25\% weight loss, relatively to the highest weigh reached.

Mice euthanasia was performed through controlled $\mathrm{CO}_{2}$ inhalation or an overdose of ketamine $(150 \mathrm{mg} / \mathrm{kg})$ and medetomidine $(2 \mathrm{mg} / \mathrm{kg}$ ) injected intraperitoneally (i.p.), followed by lethal blood collection (performed after confirmation of anesthesia) and thoracotomy.

All experiments were performed in accordance with the recommendations of the European Convention for the Protection of Vertebrate Animals Used for Experimental and Other Scientific Purposes (ETS 123) and 86/609/EEC Directive and Portuguese rules (DL 129/92). The animal facility and people directly involved in animal experiments were certified by the Portuguese regulatory entity - Direção Geral de Alimentação e Veterinária (DGAV); the animal experimental protocols were approved by DGAV (\# 015584), or by the Institutional Animal Care and Use Committee at the University of Massachusetts Medical School (Animal Welfare A3306-01), using the recommendations from the Guide for the Care and Use of Laboratory Animals of the National Institutes of Health and the Office of Laboratory Animal Welfare.

\section{Single Cell Suspensions}

Spleens, thymi and BM were collected and processed individually. Single cell suspensions were obtained from thymi and spleens by gentle mechanical dissociation in complete DMEM (cDMEM - DMEM supplemented with 10\% heatinactivated FBS, $10 \mathrm{mM}$ HEPES, $1 \mathrm{mM}$ sodium pyruvate, 2 $\mathrm{mM} \mathrm{L}$-glutamine, $50 \mathrm{mg} / \mathrm{ml}$ streptomycin, and $50 \mathrm{U} / \mathrm{ml}$ penicillin, all from Invitrogen Life Technologies); and from femurs by gentle flush of the BM with cDMEM. Red blood cells were lysed using a hemolytic solution $\left(155 \mathrm{mM} \mathrm{NH}_{4} \mathrm{Cl}, 10\right.$ $\mathrm{mM} \mathrm{KHCO}_{3}, \mathrm{pH}$ 7.2) for $4 \mathrm{~min}$ at room temperature, and cells were re-suspended in cDMEM. The number of viable cells was counted by trypan blue exclusion using a hemocytometer.

\section{Flow Cytometry}

One million cells were stained per panel for flow cytometry analysis. For BM analysis, cells were stained with FITCconjugated anti-lineage markers [anti-CD3 (145-2C11), antiCD4 (RM4-5), anti-CD8 (53-6.7), anti-CD19 (6D5), anti-B220 (RA3-6B2), anti-CD11b (M1/70), anti-CD11c (N418), anti-NK1.1 (PK136), anti-Gr1 (RB6-8C5), anti-TER119 (TER119)], PEconjugated anti-cKit (2B8), PerCP-Cy5.5-conjugated anti-Scal (D7), PE-Cy7-conjugated anti-IL7R $\alpha$ (A7R34), APC-conjugated anti-Flt3 (A2F10), APC-Cy7-conjugated anti-CD48 (HM48-1) and BV421-conjugated anti-CD150 (TC15-12F12.2). For thymocyte analysis, cells were labeled with distinct combinations of the following antibodies: FITC, PerCP-Cy5.5, BV510 or V500- conjugated anti-CD8 (53-6.7), PerCP-Cy5.5-conjugated antiCD24 (M1/69), PE or APC-conjugated anti-CD3 (145-2C11), PE-Cy7 or APC-Cy7-conjugated anti-CD44 (IM7), APC-Cy7 or V450-conjugated anti-CD4 (RM4-5), Alexa647-conjugated antiactive caspase-3 (C92-605) and APC-conjugated Annexin V. For live/dead cell analysis, propidium iodide (PI; Sigma-Aldrich, Germany) was added at a final concentration of $1 \mathrm{mg} / \mathrm{ml}, 15$ min before acquisition on the flow cytometer. For splenocyte analysis, cells were labeled with the following antibodies: FITCconjugated anti-CD11b (M1/70), APC-conjugated anti-CD3 (1452C11), APC-Cy7-conjugated anti-CD19 (6D5), V450-conjugated anti-CD4 (RM4-5) and V500-conjugated anti-CD8 (53-6.7). All antibodies were purchased from BioLegend (San Diego, CA, USA) except the V450-conjugated anti-CD4 (RM4-5), the V500conjugated anti-CD8 (53-6.7) and the Alexa647-conjugated antiactive caspase-3 (C92-605), which were obtained from BD Biosciences (San Jose, CA, USA). Acquisition was performed on a LSRII flow cytometer (equipped with 3 lasers: blue - 5 detectors, red - 2 detectors and violet - 6 detectors) using BD FACSDiva software v6.0 (Becton and Dickinson, NJ, USA), or on a MACSQuant flow cytometer (Miltenyi Biotec, Germany; equipped with 3 lasers: blue - 5 detectors, red - 2 detectors and violet - 2 detectors). Data were analyzed using FlowJo 10.7.1 (BD Biosciences).

\section{Thymic Transplant}

Thymi were aseptically removed from uninfected RAGKO or from WT mice infected for 70 days with M. avium 25291. Thymi were maintained in cDMEM for no longer than 20 min until being transplanted under the kidney capsule of WT CD45.1 mice (anesthetized with $200 \mathrm{mg} / \mathrm{Kg}$ xylazine hydrochloride and 200 $\mathrm{mg} / \mathrm{Kg}$ ketamine hydrochloride, administered i.v.). One thymic lobe from uninfected RAGKO mice and one thymic lobe from infected WT mice were transplanted to the same WT CD45.1 mouse (one on each kidney). Mice were euthanized 4 weeks after transplant, and the transplanted thymi analyzed by flow cytometry.

\section{BM Adoptive Transfer}

Single-cell suspensions of pools of BM cells were prepared from uninfected or from 70 days M. avium 25291 infected WT or IFN $\gamma \mathrm{KO}$ mice. BM progenitor cells were purified from each suspension using the Lineage Cell Depletion Mouse Kit microbeads (Miltenyi Biotec). Magnetic separation was performed with an autoMACS separator (Miltenyi Biotec). After purification, viable cells were counted by trypan blue exclusion using a hemocytometer; purity was confirmed by flow cytometry stain using FITC-conjugated anti-lineage markers and PE-conjugated anti-cKit. Cells (1-1.5 x 106) were transferred i.v. to RAGKO or iNOS.RAG.2KO mice treated with Busulfan (0.6 mg/mouse) administered i.p. $24 \mathrm{~h}$ before. Recipient mice received prophylactic antibiotic treatment ad libitum [2,5\% of Bactrim (40 mg trimethoprim and $200 \mathrm{mg}$ sulfametoxazol) in drinking water] for 5 days (treatment finished 2 days before BM transfer). Mice were euthanized 4 weeks after cell transfer and the recipient thymus and spleen were analyzed by flow cytometry. 


\section{Measurement of Corticosterone Serum Levels}

To obtain the serum concentration of basal corticosterone levels, blood samples were collected at 9 am ( $1 \mathrm{~h}$ after lights are on at the animal facility) from a venous incision at the tip of the tail during a period not exceeding $2 \mathrm{~min}$ for each mouse (to avoid corticosterone sera level increase due to handling). Sera were isolated by centrifugation and stored at $-80{ }^{\circ} \mathrm{C}$. Corticosterone levels were determined using Corticosterone ELISA kit (ENZO life sciences, Inc., NY, USA) according with manufacturer's instructions.

\section{Real Time-Quantitative PCR Analysis}

Total RNA was extracted from 1 thymic lobe (except for $70 \mathrm{dpi}$, where the whole thymus was used due to severe atrophy) and from $\mathrm{BM}$ cells recovered from two femurs per mouse, using TRIzol ${ }^{\mathrm{TM}}$ Reagent (Invitrogen, Carlsbad, CA, USA). RNA was quantified using NanoDrop 2000 (Thermo Scientific, MA, USA), and $1 \mu \mathrm{g}$ was run on a $1 \%$ agarose gel to check for RNA integrity. Complementary DNA (cDNA) was synthesized using iScriptTM Advanced cDNA Synthesis Kit (Bio-Rad Laboratories, CA, USA), according to manufacturer's instructions. Real Time-quantitative PCR (RT-qPCR) was performed using the SsoFast EvaGreen Supermix or the SsoFast EvaGreen low ROX (Bio-Rad Laboratories, CA, USA) and the primer pairs described in Supplementary Table 1. Expression of target and housekeeping genes was analyzed on CFXTM Manager (Bio-Rad Laboratories) using the "Gene Study" function or on the 7500 Fast Real-Time PCR System using the 7500-software version 2.3 (Applied Biosystems by Life Technologies) and exported to Microsoft Excel for further calculations. 18S rRNA, Gapdh and Hprt were used as housekeeping genes. The expression of Gapdh did not comply with the requirements to be used as housekeeping gene and was excluded from the analysis. Target genes expression presented in graphs are relative to the expression of the housekeeping genes $18 S$ rRNA and Hprt.

\section{Statistical Analysis}

Results were expressed as mean or mean $+\mathrm{SD}$, unless otherwise said. Prism 8.4.3 (GraphPad, CA, USA) was used for all the statistical analysis. Variables' normality was accessed by Kolmogorov-Smirnov test. Tests were used as indicated in figure legends: two-tailed unpaired $t$-test, Mann-Whitney test, two-tailed ratio paired $t$-test, ordinary one-way ANOVA followed by Dunnett's multiple comparisons test, or 2-way ANOVA followed by Tukey's multiple comparisons test. Differences between groups were considered statistically significant for $p$-values $<0.05$.

\section{RESULTS}

\section{Increased Proportion of LSK Cells in the BM of Mice Infected With $M$. avium Strain 25291 Requires IFN $\gamma$ but Not iNOS}

The differentiation of new $\mathrm{T}$ cells is dependent on the continuous seeding of BM T cell precursors in the thymus. We hypothesized that M. avium infection causes alterations on $\mathrm{T}$ cell precursors which contribute to infection-induced thymic atrophy. To test this hypothesis, we analyzed the BM from WT mice infected with M. avium strain 25291, which induces premature thymic atrophy, or with $M$. avium strain 2447, which does not cause premature thymic atrophy. Additionally, BM from mice lacking IFN $\gamma$ (IFN $\gamma \mathrm{KO}$ ), iNOS (iNOSKO) or the signaling of IFN $\gamma$ in $\mathrm{M} \phi$ (MIIG), crucial factors that contribute to M. avium strain 25291 infection-induced thymic atrophy (10), were also analyzed after infection.

We observed that the bacterial load in the BM from mice infected with strain 25291 is higher compared to that of mice infected with strain 2447, reaching a 5-log difference at 70 days post-infection (dpi; Figure 1A). At 80 dpi with M. avium strain 25291, mice lacking IFN $\gamma$ have similarly high bacterial load in their BM compared to WT mice (Figure 1B), and iNOSKO mice have a 2-log lower bacterial load than WT mice (Figure 1C), in agreement with the bacterial load in the liver, spleen, lung and thymus $(10,11)$. Infection of WT mice with strain 25291 leads to decreased number of total BM cells (Figure 1D), as previously described (46). On the other hand, the total number of BM cells from WT mice infected with strain 2447 does not differ from those of uninfected animals through 80 dpi (Figure 1D).

To examine $T$ cell precursors in the BM during infection with $M$. avium strain 25291, we analyzed the different BM cell populations by flow cytometry. Hematopoietic stem cells (HSC), which are part of the LSK (Lineage $\mathrm{Scal}^{+} \mathrm{CKit}^{+}$; see gating strategy, Figure 2A) population, possess the capacity for self-renewal and can give rise to all mature blood cell types (47). In comparison to uninfected mice, animals infected with $M$. avium strain 2447 have a transient increase in LSK cells percentage at $30 \mathrm{dpi}$, which return to normal levels over time. In contrast, in mice infected with the high virulence strain 25291, there is a sustained increase in the percentage of LSK cells throughout the time course evaluated (Figure 2B). Consistent with the described induction of LSK cell expansion mediated by $\operatorname{IFN} \gamma(39)$, there is no alteration on LSK cells percentage in IFN $\gamma \mathrm{KO}$ mice infected with $M$. avium strain 25291 up to 80 dpi (Figure 2C). To understand if the increase in the percentage of LSK population is due to a direct effect of IFN $\gamma$, or another mechanism dependent on M $\phi$ activation, MIIG mice were infected. At 80 dpi, MIIG mice show increased LSK cells percentage when compared to uninfected mice. However, the percentage of LSK cells is lower on MIIG in comparison to WT mice (Figure 2D). As for iNOSKO mice, we show that the percentage of LSK cells increase upon infection with strain 25291 (80 dpi), similarly to WT mice (Figure 2E). Together, these results show that the increase on LSK cells percentage during infection by M. avium strain 25291 is partially dependent on IFN $\gamma$ induced activation of $\mathrm{M} \phi$ but not on iNOS production. Finally, infection by $M$. avium strain 25291 leads to a dramatic decrease in the frequency of long term (LT)-HSC and short term (ST)-HSC (Figures 2F, G).

\section{Increased LMPP and Decreased CLP in the BM After Infection With $M$. avium 25291 Is Independent on IFN $\gamma$ and iNOS}

There are two BM cell populations described as able to differentiate into $\mathrm{T}$ cells in vitro and in vivo (48): (i) the lymphoid-primed multipotent progenitors (LMPP), which is a subpopulation of LSK 

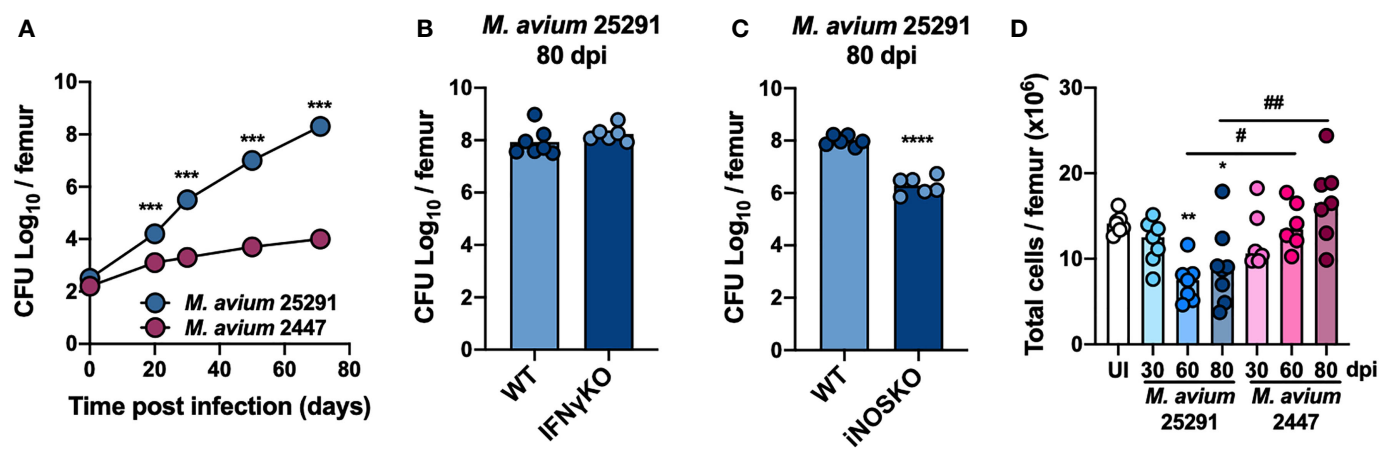

FIGURE 1 | Bacteria load in the BM is higher after infection with M. avium strain 25291 than with strain 2447. (A) Representative kinetics of M. avium infection in the BM of WT mice infected with strains 25291 (blue) or 2447 (pink). (B, C) Bacterial load in the BM from WT, IFN\}KO and iNOSKO mice infected with M. avium strain 25291 for 80 days. At each time-point of infection, groups were compared by two-tailed unpaired $t$-test and were marked as: ${ }^{\star \star \star} p<0.001$; ${ }^{\star \star \star \star} p<0.0001$. (D) Total number of BM cells per femur of uninfected WT mice (white), or after infection with M. avium strain 25291 (blue) or strain 2447 (pink) at 30,60 and 80 dpi. The groups of infected mice, at the several time-points, were compared with the uninfected mice by ordinary one-way ANOVA followed by Dunnett's multiple comparisons test and were marked as: ${ }^{*} p<0.05$; ${ }^{* *} p<0.01$; comparisons between infected groups were performed by 2 -way ANOVA followed by Tukey's multiple comparisons test, and marked as: ${ }^{*} p<0.05$, $\# p<0.01$. Data represent the mean \pm SD $(\mathbf{A})$ or mean (B-D) from 5 to 8 mice per group, from one of two independent experiments. UI stands for uninfected. dpi stands for days post infection.

cells that express Flt3 ( Lin $^{-}$IL-7R $\alpha^{-} \mathrm{Scal}^{+} \mathrm{cKit}^{+} \mathrm{Flt}^{+}{ }^{+}$); and (ii) the common lymphoid progenitors (CLP), which are $\mathrm{Lin}^{-} \mathrm{IL}-7 \mathrm{R} \alpha^{+}$ $\mathrm{Sca}^{\text {int }}{ }^{\mathrm{CKit}}{ }^{\text {int }} \mathrm{Flt}^{+}{ }^{+}$. Compared to uninfected mice, the percentage of LMPP (see gating strategy, Figure 3A) is increased after infection with M. avium strain 25291, but not with strain 2447 (Figure 3B). We also observed an increase in the number of LMPP with both $M$. avium strain 25291 and 2447 that is sustained only for strain 25291 (Supplementary Figure 1A). The percentage and number of LMPP is not different in M. avium strain 25291 infected IFN $\gamma \mathrm{KO}$ (Figure 3C and Supplementary Figure 1B), MIIG (Figure 3D and Supplementary Figure 1C) and iNOSKO (Figure 3E and Supplementary Figure 1D) mice comparing with WT infected mice. These results show that the increased proportion of LMPP does not require IFN $\gamma$ nor iNOS expression and thus is not sufficient to cause infection-induced thymic atrophy.

We observed an obvious decrease in both percentage and number of CLP (see gating strategy, Figure 3F) in WT mice infected with M. avium strain 25291, a change that is also observed in mice infected with the low virulence strain 2447 (Figure 3G and Supplementary Figure 1E). We also observed a reduction in the percentage of CLP in M. avium 25291 infected IFN $\gamma \mathrm{KO}$ mice (Figure 3H). But the decrease in the percentage of CLP upon infection with strain 25291 is dependent on the activation of M $\phi$ by IFN $\gamma$, since MIIG mice have the same percentage of CLP as uninfected mice (Figure 3I). iNOSKO mice infected with $M$. avium strain 25291 have a reduction in the percentage of CLP similar to WT infected mice (Figure 3J). The number of CLP is reduced in IFN $\gamma \mathrm{KO}$, MIIG and iNOSKO mice, as observed in WT mice, at $80 \mathrm{dpi}$ (Supplementary Figures 1F-H). These results show that IFN $\gamma$ and the production of NO, are not required for the CLP alterations upon M. avium strain 25291 infection.

To assess other molecules that might be involved in the observed alterations in BM cell populations, we analyzed the expression of genes previously described to affect the BM in various infection/inflammation models. We observed that
interleukine-6(Il6) expression is increased in WT mice infected with M. avium strain 25291 compared to uninfected mice but not in infected iNOSKO mice (Supplementary Figure 2A). No significant differences were observed after infection in the expression of glucocorticoid receptor $(G r)$ and tumor necrosis factor $(\operatorname{Tn} f)$ in the BM (Supplementary Figures 2B, C).

\section{BM Cells From WT Mice Infected With $M$. avium 25291 Poorly Reconstitute Thymi From RAGKO Mice}

Since the observed reduction on the percentage and number of CLP is independent of IFN $\gamma$ and iNOS, this alteration was excluded as the mechanism responsible per se for $M$. avium-induced thymic atrophy; still it might synergize with other mechanisms yet to be identified. To investigate if alterations in BM precursors contribute to $M$. avium infection-induced thymic atrophy, we transferred lineage negative BM cells from uninfected or 70 days infected WT or IFN $\gamma \mathrm{KO}$ mice, into RAGKO recipients. Recipient mice were euthanized 4 weeks later. Mice receiving BM cells from WT mice infected with M. avium 25291 present an overall weaker thymic reconstitution than mice receiving $\mathrm{BM}$ cells from uninfected mice or from IFN $\gamma \mathrm{KO}$ infected and uninfected mice (Figure 4A). The observation that $\mathrm{BM}$ from infected IFN $\gamma \mathrm{KO}$ mice has the same ability to reconstitute the thymus as WT uninfected reveals that the reduced ability to reconstitute the thymus of infected WT BM cells is not dependent on the altered percentage/number of CLP and LMPP. In agreement with the data from thymic reconstitutions, a lower number of total splenocytes, including total and both $\mathrm{CD}^{+}$ and $\mathrm{CD}^{+} \mathrm{T}$ cells, were recovered from mice receiving $\mathrm{BM}$ from infected WT mice, compared to mice receiving BM cells from uninfected WT or IFN $\gamma \mathrm{KO}$ infected mice (Figure 4B).

To test if the reconstitution of BM precursor cells from WT infected mice is inhibited by NO production in the recipient thymus, lineage negative BM cells from WT mice, uninfected or M. avium 25291 infected (70 dpi) were transferred to 

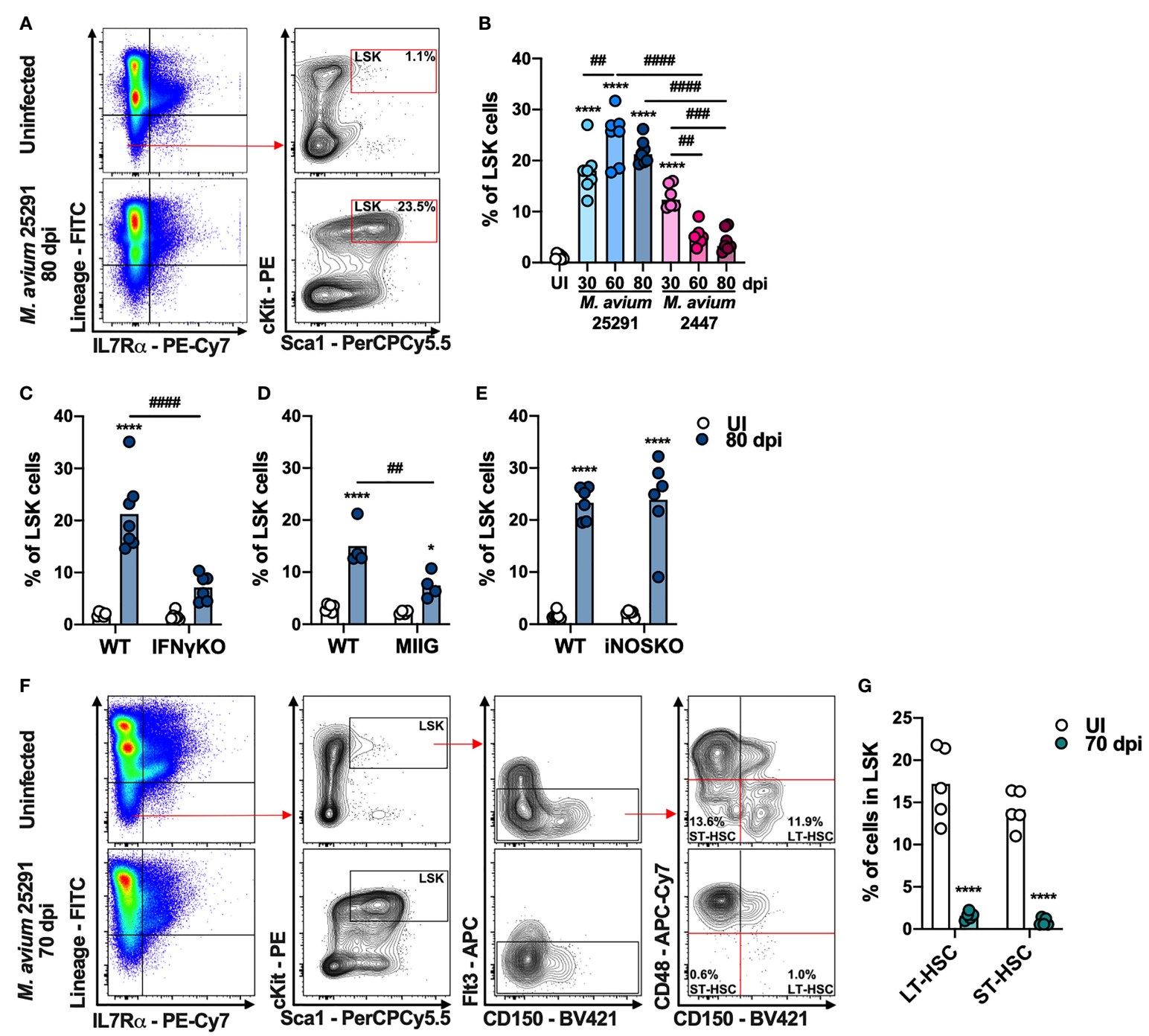

FIGURE 2 | Mice infected with M. avium strain 25291 have higher percentage of LSK cells in an IFN $\gamma$-dependent and iNOS-independent manner. (A) Schematic representation of the gating used to identify LSK cell population in the BM from uninfected WT mice (top), or infected for 80 days with M. avium 25291 (bottom). Total BM cells were previously selected eliminating doublets and debris. (B) Percentage of LSK cells from uninfected WT mice (white) or after infection with M. avium strain 25291 (blue) or strain 2447 (pink) at 30, 60 and 80 dpi. Comparisons between infected and uninfected mice were performed using ordinary one-way ANOVA followed by Dunnett's multiple comparisons test, and marked as: ${ }^{\star \star \star \star} p<0.0001$; comparisons between all the infected groups were performed using a 2-way

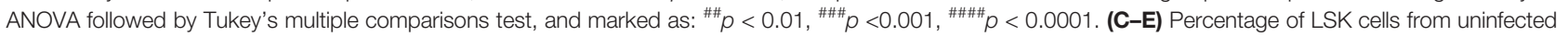
(white) or infected for 80 days with M. avium 25291 (blue) of WT, IFNYKO, MIIG or iNOSKO mice. Groups were compared using 2-way ANOVA followed by Tukey's multiple comparisons test; statistical differences between uninfected and infected mice were marked as ${ }^{*} p<0.05$ and ${ }^{* \star *} p<0.0001$; and between infected groups

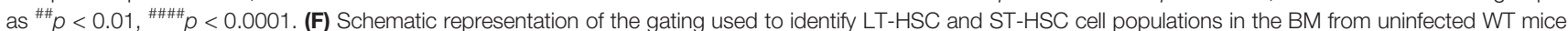
(top), or infected for 70 days with M. avium 25291 (bottom) (G) Percentage of LT-HSC and ST-HSC cells in WT uninfected (white) or after 70 days of infection with M. avium 25291 (teal). Comparisons between infected and uninfected were performed by two-tailed unpaired $t$-test and marked as ${ }^{\star \star \star \star} p<0.0001$. Bars represent the mean from 4 to 8 mice per group from one of two independent experiments. UI stands for uninfected. dpi stands for days post infection.

iNOS.RAG.2KO recipients. Irrespective of donor BM cells being from infected or uninfected mice, no differences between RAGKO and iNOS.RAG.2KO recipient mice were observed when comparing the reconstitution of the thymus (Figure $4 \mathrm{C}$ ), and the $\mathrm{T}$ cell pool in the spleen (Figure 4D).

The recruitment and entry of $\mathrm{BM} \mathrm{T}$ cell precursors to the thymus is mediated by chemokines expressed by thymic epithelial cells (TEC), such as CCL21, CCL25 and CXCL12, as well as P-selectin. These molecules are recognized by their receptors in precursor cells, such as CCR7, CCR9, CXCR4 and PSGL1. The analysis of the expression of these molecules revealed increased expression of Ccl25, Cxcl12 and P-selectin in the thymus (Figure 4E), and decreased expression of $\mathrm{Ccr} 7$ in $\mathrm{BM}$ cells (Figure 4F) upon M. avium strain 25291 infection.

Overall, these results show that BM T cell precursors from mice infected for 70 days with M. avium 25291 have a lower 


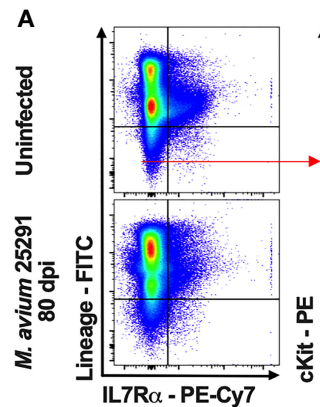

B

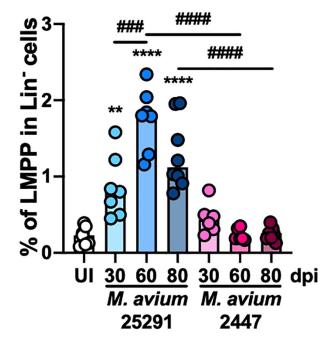

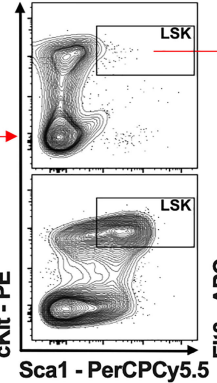

C

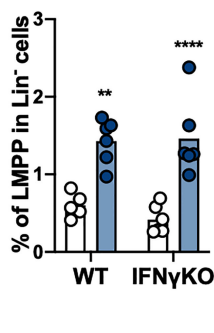

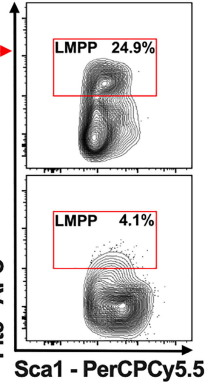

D

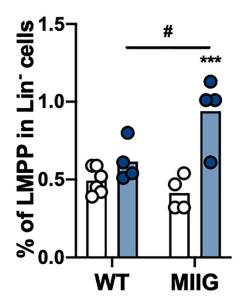

E

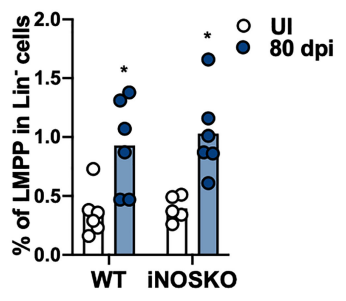

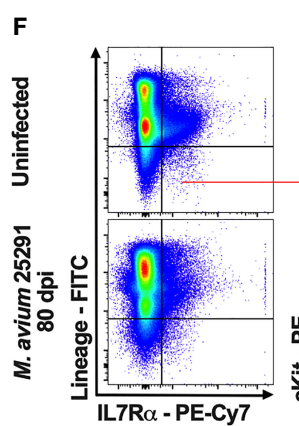

G

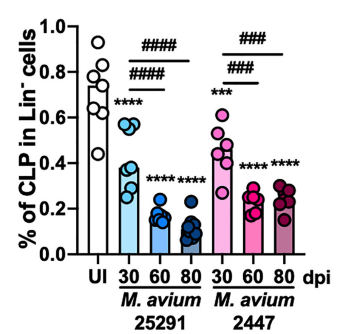

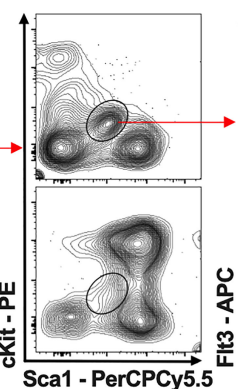

$H$

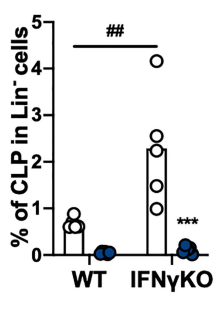

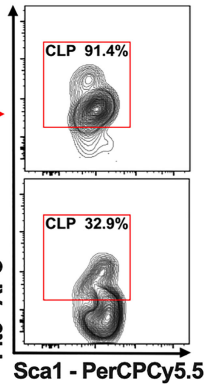

I

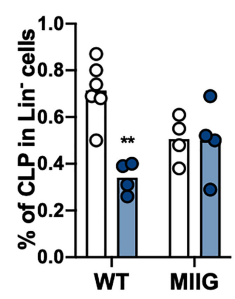

$\mathbf{J}$

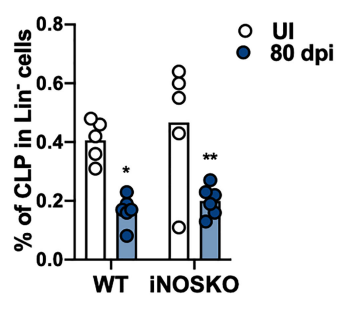

FIGURE 3 | The increase on the percentage of LMPP and the decrease on the percentage of CLP in mice infected with M. avium 25291 is independent on IFN $\gamma$ and iNOS. (A) Schematic representation of the gating used to select LMPP population in the BM from uninfected WT mice (top), or infected for 80 days with M. avium 25291 (bottom). Total BM cells were previously selected eliminating doublets and debris. (B) Percentage of LMPP in the lineage negative population from uninfected (white) WT mice or infected with M. avium strain 25291 (blue) or strain 2447 (pink) at 30, 60 and 80 dpi. (C-E) Percentage of LMPP in the lineage negative population from uninfected (white) or infected for 80 days with M. avium 25291 (blue), WT, IFN $\gamma$ KO, MIIG or iNOSKO mice. (F) Schematic representation of the gating used to select CLP population in the BM from uninfected WT mice (top), or infected for 80 days with M. avium 25291 (bottom). Total BM cells were selected eliminating doublets and debris. (G) Percentage of CLP in the lineage negative population from uninfected WT mice (white), or infected with M. avium strain 25291 (blue) or strain 2447 (pink) at 30, 60 and 80 dpi. (H-J) Percentage of CLP in the lineage negative population from uninfected (white) or infected for 80 days with M. avium 25291 (blue), WT, IFNүKO, MIIG or iNOSKO mice. In all graphs, bars represent the mean from 4 to 8 mice per group, from one of two independent experiments. In B and $\mathrm{G}$ comparisons between infected and uninfected mice were evaluated by ordinary one-way ANOVA followed by Dunnett's multiple comparisons test and marked as: ${ }^{* \star} p<0.01,{ }^{\star \star *} p<0.001,{ }^{* \star * *} p<0.0001$; comparisons between infected groups were evaluated by 2-way ANOVA followed by Tukey's multiple comparisons test and marked as: ${ }^{\# \#} p<0.001, " \# \# \#<0.0001$. In (C-E, H-J), comparisons were evaluated by 2-way ANOVA followed by Tukey's multiple comparisons test and marked as: ${ }^{*} p<0.05,{ }^{\star *} p<0.01,{ }^{* \star *} p<0.001,{ }^{* \star * *} p<0.0001$ for comparisons

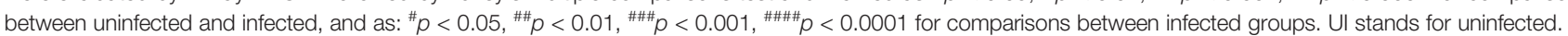
dpi stands for days post infection. 

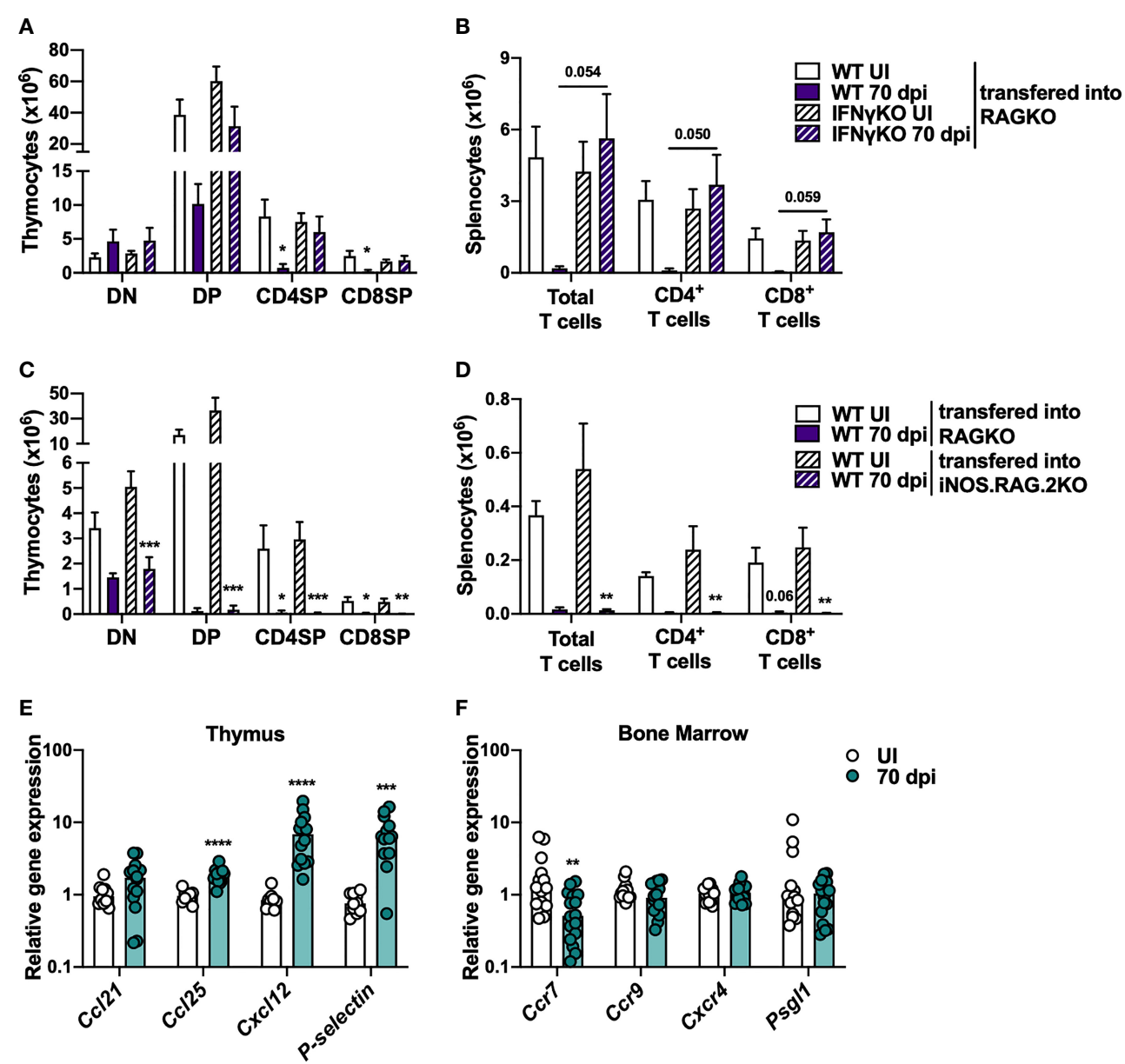

FIGURE 4 | Overall decreased ability to reconstitute thymi from RAGKO mice by BM cells from M. avium strain 25291 infected mice is IFNy-dependent. (A, B) RAGKO mice were reconstituted with lineage negative BM cells from uninfected (white) or 70 days M. avium 25291 infected (purple) WT (solid) or IFNyKO mice (dashed). (A) Number of the four main thymocyte populations (DN, DP, CD4SP and CD8SP). (B) Number of splenic total T cells, and of CD4 $4^{+}$and CD8 $8^{+}$T cells. (C, D) RAGKO (solid) or iNOS.RAG.2KO (dashed) mice were reconstituted with lineage negative BM cells from uninfected (white) or 70 days M. avium 25291 infected (purple) WT mice. (C) Number of cells from the four main thymocyte populations. (D) Number of splenic T cells and its subpopulations. RNA expression levels in WT mice uninfected (white) or infected with M. avium strain 25291 for 70 days (teal). (E) Expression in the thymus of the chemokines CCl21, CCl25 and CxCl12, and of P-selectin. (F) Expression in the BM of the chemokines receptors Ccr7, Ccr9 and Cxcr4, and of Psgl1. In (A-D) bars represent the mean + SEM from 3 to 6 mice per group from one of two independent experiments. Comparisons were performed using 2-way ANOVA followed by Tukey's multiple comparisons test, and marked between infected and uninfected mice as: ${ }^{*} p<0.05,{ }^{* *} p<0.01$, ${ }^{* \star *} p<0.001$. For $p$-values between [0.05; 0.10], their values are represented in the graphs. In $E$ and $F$ bars represent the median from 10 to 16 mice per group from two pooled independent experiments. Comparisons between infected and uninfected were performed by two-tailed unpaired $t$-test or Mann-Whitney test, according to normality, and marked as ${ }^{\star \star} p<0.01,{ }^{\star \star \star} p<0.001,{ }^{\star \star \star \star} p<0.0001$. Ul stands for uninfected. dpi stands for days post infection. DN stands for double negative. DP stands for double positive. SP stands for single positive.

ability to reconstitute mice lacking $\mathrm{T}$ cells in a process dependent on the expression of IFN $\gamma$ on BM cells but independent of NO production by the recipient thymus. The data presented here support the hypothesis that infection-induced BM cells alterations play a relevant part on thymic atrophy caused by M. avium infection.

\section{IFN $\gamma$ and iNOS Expression Is Associated With a More Inflammatory Profile in the Thymus After Infection}

The observation that IFN $\gamma \mathrm{KO}$ and iNOSKO mice have the same alterations on the percentage/number of $\mathrm{BM} \mathrm{T}$ cell precursors as WT mice after infection, but no premature thymic atrophy (10), suggests that these alterations per se are insufficient to cause premature thymic atrophy. Therefore, we hypothesized that IFN $\gamma$ and NO production have a direct impact in the thymus that synergizes with alterations in the BM precursor cells. To investigate this hypothesis, the expression of inflammatory molecules in the thymus of WT, IFN $\gamma \mathrm{KO}$ and iNOSKO mice was evaluated after infection with $M$. avium strain 25291. We observed lower expression of iNos in the thymus of infected IFN $\gamma \mathrm{KO}$ mice compared to infected WT mice (Figure 5A), while Ifng expression is increased upon infection, independently of iNOS expression (Figure 5B). In respect to 

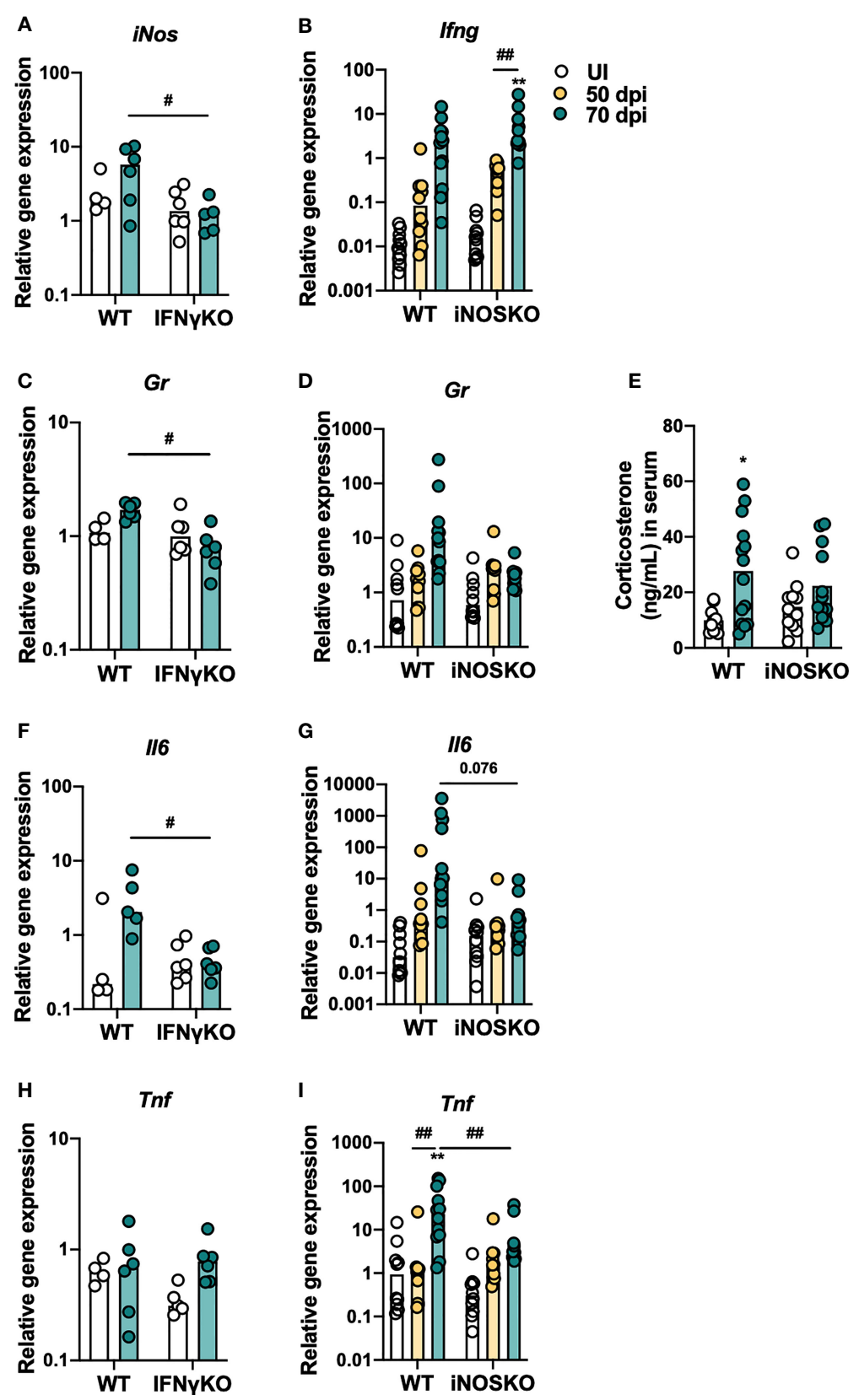

FIGURE 5 | M. avium strain 25291 infected IFN $\gamma K O$ and iNOSKO mice present a milder inflammatory profile in the thymus than WT infected mice. RNA expression levels in the thymus of WT, IFNYKO and iNOSKO mice uninfected (white) or infected with M. avium strain 25291 for 50 (yellow) or 70 (teal) days. (A) iNos; (B) Ifng; (C, D) Gr; (F, G) /16; and (H, I) Tnf. (E) Basal corticosterone levels in the serum of WT and iNOSKO mice uninfected (white) or infected with M. avium strain 25291 for 70 days (teal). Bars represent the median for all graphs except for $\mathbf{( E )}$ where mean is represented; for $(\mathbf{A}, \mathbf{C}, \mathbf{F}, \mathbf{H})$ from 4 to 6 mice per group from one experiment; for (B, D, E, G, I) from 10 to 12 mice per group from two pooled independent experiments. Statistically significant differences were accessed by 2 -way ANOVA followed by Tukey's multiple comparisons test and marked between uninfected and infected groups as: ${ }^{\star} p<0.05$, ${ }^{\star \star} p<0.01$; and between infected groups as: ${ }^{\#} p<0.05, " \# p<0.01$. For $p$-values between [0.05; 0.10], their values are represented in the graphs. Ul stands for uninfected. dpi stands for days post infection.

Gr expression, no clear differences were observed upon infection with M. avium strain 25291 (Figures 5C, D), although WT, but not iNOSKO infected mice, show higher serum levels of corticosterone (Figure 5E). Infection induces the expression of $I l 6$ in WT mice but not in IFN $\gamma \mathrm{KO}$ and
iNOSKO mice (Figures 5F, G). We also observed a tendency for increased Tnf expression in the thymus of WT, not present in iNOSKO mice (Figures 5H, I), despite high variability for this particular gene within groups and between experiments. 


\section{Thymic Stroma From M. avium 25291 Infected Mice Is Unable to Support Optimal T Cell Differentiation}

To determine if alterations in the thymic stroma (considered here as composed by TECs, antigen presenting cells and the overall milieu) contribute to infection-induced thymic atrophy, we investigated the ability of thymi from infected animals to support thymocyte differentiation when BM cells from uninfected mice are provided. Thus, atrophied thymi from infected WT mice (CD45.2) and from uninfected RAGKO mice (CD45.2), were transplanted under the kidney capsules of uninfected WT CD45.1 mice (i.e., one thymus on each kidney of the same recipient mouse; Figure 6A). Four weeks after transplant mice were euthanized and the four main thymocyte populations within $\mathrm{CD} 45.1^{+}$cells were analyzed in the transplanted thymi and in the endogenous thymi of recipient mice (Figure 6B). Transplanted thymi from infected WT mice show lower percentage of total thymocytes with origin in the recipient mice $\left(\mathrm{CD} 45.1^{+}\right)$, when compared with transplanted thymi from uninfected RAGKO mice. Still, precursor cells from recipient mice are recruited and replace most of the donor thymocytes in the transplanted thymi from infected WT mice, as the majority of cells recovered are CD $45.1^{+}$(Figure 6C). The percentage of the thymocyte populations in the transplanted WT infected thymi are significantly distorted, while that in the transplanted RAGKO thymi are similar to the ones observed in the endogenous thymi (Figure 6D). The absolute number of each thymocyte population is higher in uninfected RAGKO thymi than in thymi from infected WT mice (Figure 6E). These results show that thymic stroma from WT mice infected with M. avium 25291 has an impaired ability to support thymocyte differentiation.

Upon 70 days of infection by $M$. avium strain 25291, a disruption of the thymic structure is observed, although there is variability between mice. While some infected WT mice maintain a compartmentalized thymic structure, with clear distinction between cortex (cTECs, stained mostly by K8) and medulla (mTECs, stained mostly by K5), others present a thymus without clear distinction of the two main compartments and densely stained for K5 and K8 (Supplementary Figure 3). However, even in infected WT mice that preserve a clear compartmentalization between cortex and medulla, there is a reduction of the cortex region, as observed in $\mathrm{K} 8$ and $\mathrm{HE}$ stains (Supplementary Figure 3).

\section{Increased Thymocyte Death in Infected Mice Is Independent of Caspase-3 Activation}

To further dissect the mechanisms that lead to infection-induced thymic atrophy we investigated thymocyte death by analyzing the incorporation of propidium iodide (PI) and binding of annexin V by thymocytes. Infection by both M. avium strains (25291 and 2447) leads to a reduction in the percentage of viable cells (Annexin $\mathrm{V}^{-} \mathrm{PI}^{-}$) and an increase in the percentage of apoptotic cells (Annexin $\mathrm{V}^{+} \mathrm{PI}^{-/ \text {low }}$ ) (Figure 7A). However, these alterations occur earlier and are of a greater magnitude in mice infected with the high virulence strain 25291 compared to mice infected with the low virulence strain 2447 (Figure 7A). Increased percentage of thymocytes undergoing necrosis/late apoptosis (Annexin $\mathrm{V}^{+} \mathrm{PI}^{\text {high }}$ ) is observed upon infection with $M$. avium strain 25291, but not with strain 2447 at 70 dpi (Figure 7A).

To understand if these alterations are dependent on IFN $\gamma$ and/or NO production, we analyzed thymocyte cell death in thymi from IFN $\gamma \mathrm{KO}$ and iNOSKO mice at $70 \mathrm{dpi}$. The reduction in the percentage of viable thymocytes, and the increase in the percentage of apoptotic thymocytes is similarly observed in IFN $\gamma \mathrm{KO}$ and iNOSKO mice, indicating that these alterations in death do not require IFN $\gamma$ or NO (Figure 7B). However, neither IFN $\gamma \mathrm{KO}$ nor iNOSKO infected mice reveal an increased percentage of necrotic/late apoptotic thymocytes in comparison to the uninfected peers (Figure 7B). With respect to the four main thymocyte populations, DP, CD4 single positive (SP) and CD8SP are the ones most affected after infection, in an IFN $\gamma$ and NO independent manner (Supplementary Figure 4AD). In infected WT mice, DP thymocytes is the only population with increased percentage of necrosis/late apoptosis, a difference not present in IFN $\gamma \mathrm{KO}$ or iNOSKO infected mice, as observed in total thymocytes (Supplementary Figure 4B).

Finally, we observed that infection with both $M$. avium strains leads to decreased percentage of active caspase- 3 positive thymocytes. In mice infected with strain 25291, this decrease is evident as early as $30 \mathrm{dpi}$, and is sustained up to $70 \mathrm{dpi}$. In contrast, the reduction in the percentage of active caspase-3 positive thymocytes is only observed at 70 dpi in mice infected with strain 2447 (Figure 7C). Upon infection with strain 25291, the percentage of active caspase-3 positive thymocytes decreases in IFN $\gamma \mathrm{KO}$ mice, though to a lower extent in comparison to WT mice (Figure 7D). These results show that the increased apoptosis occurring in thymocytes after infection is independent of caspase- 3 activation. Additionally, the reduction in the percentage of active caspase- 3 positive thymocytes is partially dependent of IFN $\gamma$ and dependent of iNOS.

\section{DISCUSSION}

Infection by $M$. avium strain 25291 results in severe thymic atrophy, a process dependent on the synergy between GC and NO produced by IFN $\gamma$ activated $M \phi(10)$. Here we investigate where and how these mediators cause $M$. avium-induced thymic atrophy

Following infection with M. avium strain 25291 we found an increase in the percentage of LSK cells in the BM which is dependent on IFN $\gamma$. Our results are coherent with previous reports describing LSK expansion during infection by $M$. tuberculosis, M. avium and Vaccinia virus $(29,30,35,39)$. To our knowledge, we report for the first time that the expansion of LSK cells during M. avium infection is at least partially dependent on $\mathrm{M} \phi$ activation by IFN $\gamma$. The experiments leading to this conclusion were those using MIIG mice, whose $\mathrm{CD}^{+} 8^{+}$cells are defective in IFN $\gamma$ signaling. Although an increase on the percentage of LSK cells can be observed at 80 dpi in MIIG mice, it does not reach the same magnitude of increase as that observed in infected WT mice. The expansion of LSK cells during $M$. tuberculosis infection has been associated with two cytokines 

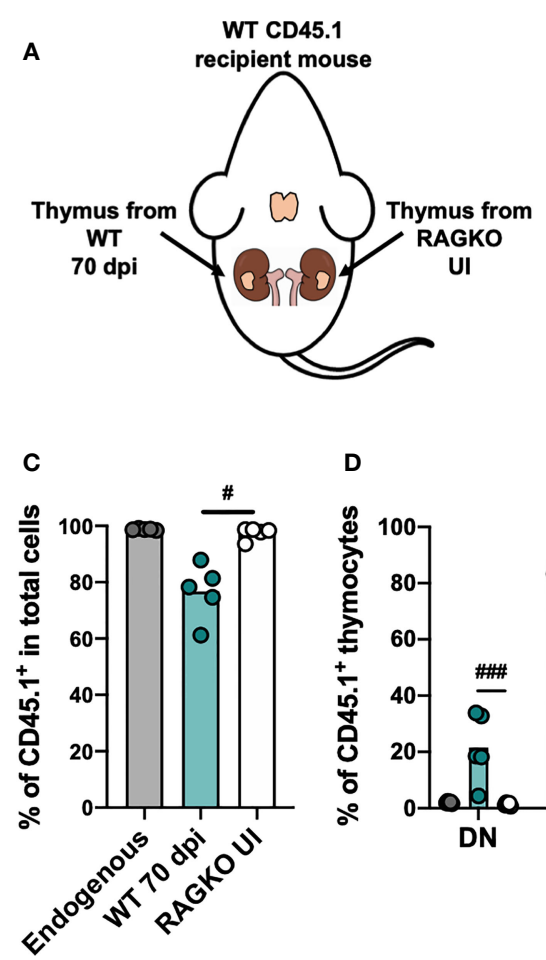

D
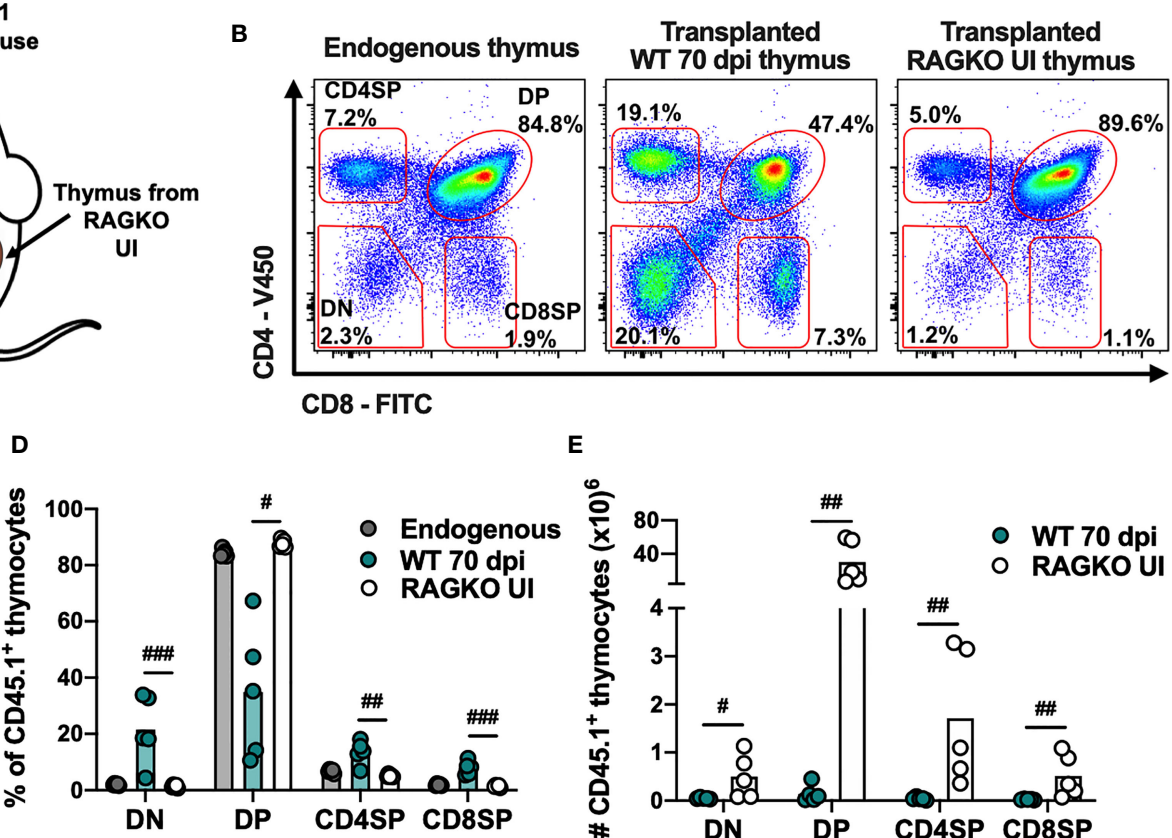

E

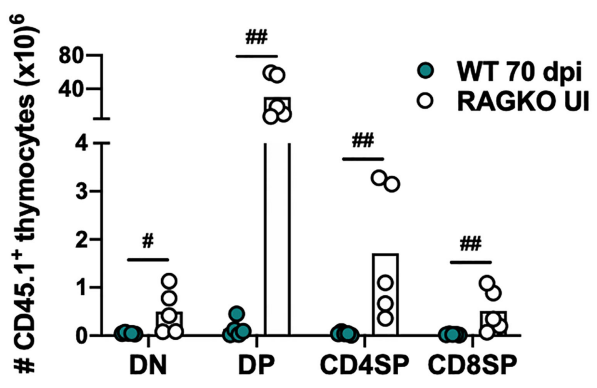

FIGURE 6 | Thymic stroma from WT mice infected with M. avium 25291 does not properly support T cell differentiation. (A) Experimental schematic representation of thymi transplant from M. avium infected WT mice (70 dpi) and uninfected RAGKO mice (both CD45.2) under the kidney capsule of WT CD45.1 recipient mice. Mice were euthanized 4 weeks after transplant. (B) Representative plots of the CD45.1 four main thymocyte populations (DN, DP, CD4SP and CD8SP) from WT CD45.1 recipient mice endogenous thymi, and from transplanted WT infected and RAGKO uninfected thymi. (C) Percentage of cells recruited from the recipient mouse $\left(C D 45.1^{+}\right)$, and (D) percentage and (E) number of cells from the four main thymocyte populations from endogenous thymi of WT CD45.1 recipient mice (grey), or from transplanted thymi from 70 days M. avium 25291 infected WT mice (teal) or uninfected RAGKO mice (white). Data represent the mean from five mice per group from one of two independent experiments. Comparisons between WT $70 \mathrm{dpi}$ and uninfected RAGKO were performed by 2-tailed ratio paired t test and

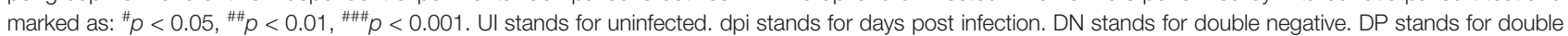
positive. SP stands for single positive.

produced by M $\phi$, TNF and IL-6 (29). We also observed an increase in the mRNA expression of these two cytokines in the BM after $M$. avium strain 25291 infection, supporting the relevance of activated $\mathrm{M} \phi$ in LSK expansion. We also show that the expansion of LSK cells is independent of iNOS, as iNOSKO infected mice present the same alteration in this BM population as WT infected mice. This shows that the expansion of LSK might be part of the mechanism leading to thymic atrophy but clearly not sufficient.

Within the LSK population, we observed a reduction in the percentage of LT-HSC and ST-HSC. HSC are very sensitive to the surrounding microenvironment and HSC alterations associated with infection and inflammation have been well described. These alterations include the reduction of the HSC pool and changes in their proliferation (30, 49-54). During chronic inflammation or chronic IFN $\gamma$ signaling there is impaired self-renewal of HSC that culminates in the reduction of this population (49). We propose that this same mechanism leads to the reduction in HSC percentage observed upon infection with M. avium strain 25291.

Regarding the most direct $\mathrm{T}$ cell precursors in the BM, CLP and LMPP, we show that M. avium infection induces an increase in the percentage and number of LMPP, which is independent of IFN $\gamma$ and iNOS. Kong et al., using a sepsis model, described an increase in the number of LMPP associated with premature thymic atrophy (41), which is consistent with our results. In contrast, the percentage and number of CLP is reduced after $M$. avium infection with either strain 25291 or 2447 , being clearly more evident for the more virulent strain 25291. This decrease of CLP is independent of IFN $\gamma$ and iNOS. Reduction of CLP was also reported after $P$. chabaudi infection, although thymic atrophy was not assessed in this study (36). In contrast, infection with Vaccinia virus, known to cause thymic atrophy, leads to an increase of CLP in the BM (35). These results indicate that directionality of the changes in this $\mathrm{BM}$ population is pathogen specific and not always associated with infectioninduced thymic atrophy.

$\mathrm{BM}$ resident $\mathrm{M} \phi$ are essential for bone marrow niche homeostasis. M $\phi$ are in close contact with HSC and contribute for the maintenance of quiescence, self-renewal and proliferation of these cells $(55,56)$. BM resident $\mathrm{M} \phi$ are also crucial for erythropoiesis (57), and it has been shown that infection with $M$. avium strain 25291 induces alterations in the production of 

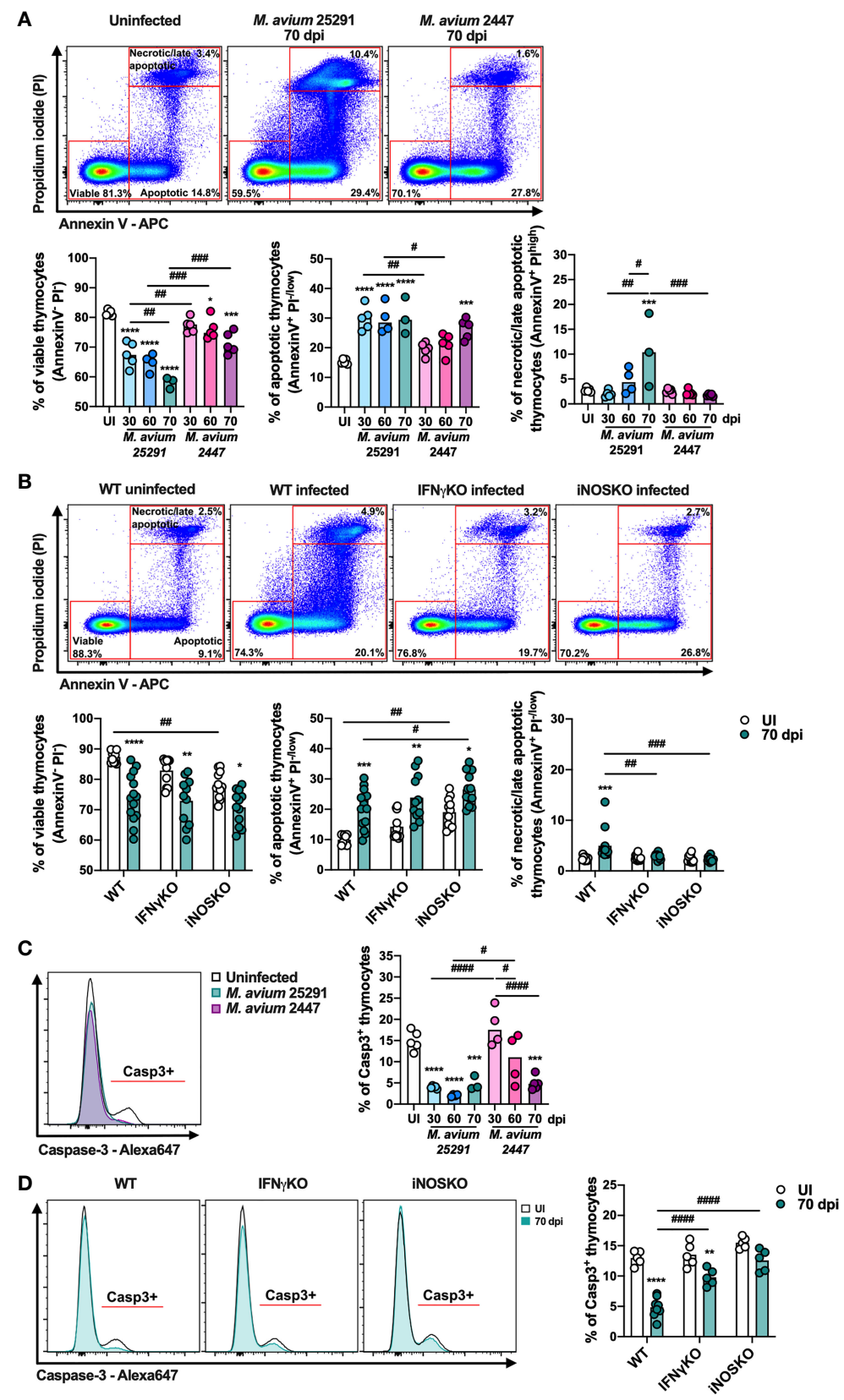

FIGURE 7 | The percentage of viable cells and of cells positive for active caspase-3 decrease after infection. (A) Representation of the gating (top) and plotting of

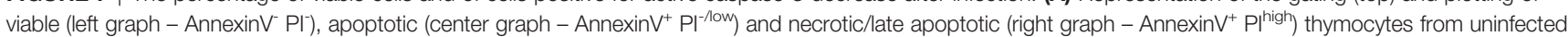
WT mice (white) or infected for 30, 60 or 70 days with M. avium 25291 (blue) or M. avium 2447 (pink). (B) Representation of the gating (top) and plotting of viable

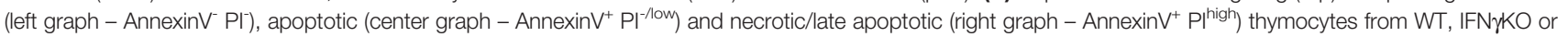
iNOSKO mice uninfected (white) or infected for 70 days with M. avium 25291 (teal). (C) Representative histogram and plotting of active caspase-3 positive thymocytes from uninfected WT mice (white) or infected for 30, 60 or 70 days with M. avium 25291 (blue) or M. avium 2447 (pink). (D) Representative histogram and plotting of caspase-3 positive thymocytes from WT, IFNYKO or iNOSKO mice uninfected (white) or infected for 70 days with M. avium 25291 (teal). For all the analysis, total thymocytes were previously selected eliminating doublets and debris. Bars represent the mean from: 3 to 5 mice per group from one experiment for (A, C); 9 to 14 mice per group from two pooled independent experiments for (B, D). In (A, C) comparisons between infected and uninfected mice were evaluated by ordinary one-way ANOVA followed by Dunnett's multiple comparisons test and marked as: ${ }^{\star} p<0.05,{ }^{\star \star \star} p<0.001$, ${ }^{\star \star \star \star} p<0.0001$; and comparisons between infected groups were evaluated by 2-way ANOVA followed by Tukey's multiple comparisons test and marked as: \#p $<0.05,{ }^{\#} p<0.01, \# \# p<0.001$, \#\#\#\#p< 0.0001. In (B, D), comparisons were performed by 2-way ANOVA followed by Tukey's multiple comparisons test, and marked as ${ }^{*} p<0.05,{ }^{* *} p<0.01,{ }^{* \star *} p<0.001$, ${ }^{* * * *} p<0.0001$ for differences between uninfected and infected, and as $\# p<0.05,{ }^{\# \#} p<0.01, \# \# p<0.001, \# \# p<0.0001$ for differences between infected groups. Ul stands for uninfected. Casp3 stands for active caspase-3. dpi stands for days post infection. 
erythrocytes and an accelerated removal of those cells from the circulation, resulting in anemia (46). Not only IFN $\gamma$ impact the $\mathrm{BM}$, other cytokines that are produced by M $\phi$, such as IL-6, TNF and type I IFN, have been also associated with disturbances in BM precursors after infection and/or inflammation $(29,34,40)$. In fact, both Il6 and Tnf mRNA expression is increased in the BM of M. avium strain 25291 infected mice. It has been described that the overproduction of IL-6 after infection with Group B Streptococcus is associated with iNOS expression (58). We also show that up-regulation of Il6 expression in the BM after infection with $M$. avium is dependent on iNOS. The activation of $\mathrm{M} \phi$ might therefore play a role in infection-induced alterations in hematopoiesis, as is suggested by the results presented here.

Unlike BM cells from uninfected WT or IFN $\gamma \mathrm{KO}$ infected mice, BM cells from M. avium strain 25291 infected WT mice have an overall impaired ability to reconstitute the thymus and the periphery of RAGKO mice. One could associate the reduced ability of BM cells from infected WT mice to reconstitute the thymus from RAGKO mice with the reduced number of CLP after infection. However, infected IFN $\gamma \mathrm{KO}$ mice show the same reduction in CLP, and the BM cells from these mice reconstitute the thymus from RAGKO mice similarly to uninfected mice. These results show that during infection by M. avium 25291, changes in the overall ability of BM cells to progress into T cell differentiation contribute to premature thymic atrophy. To our knowledge, only two reports associate BM defects with premature thymic atrophy. One of them shows that sepsisinduced thymic atrophy is associated with a dramatic decrease in early thymic precursors (ETP) as a consequence of impaired migration of progenitors from the $\mathrm{BM}$ to the thymus, and the inability of $\mathrm{BM}$ progenitors to commit to the lymphoid lineage (41). A possibility is that infection might impair the migration and entry of BM T cell precursors into the thymus. Although we showed before a reduction on the number of ETPs in the thymus of infected mice (10), we saw no obvious differences on the expression of chemokine receptors responsible for the migration of BM T cell precursors, Ccr9 and Cxcr4, nor on Psgll that is needed for the entry of the precursors in the thymus, in BM cells from infected WT mice. Only the expression of the receptor Ccr7 is decreased after infection, although no alterations were observed on the expression in the thymus of its chemokine ligand $\mathrm{Ccl} 21$. This is in contrast with a previous report on sepsisinduced thymic atrophy, in which the authors show a downregulation of the expression of CCR7, CCR9 and PSGL1 (41).

The severe alterations in the BM during $M$. avium strain 25291 infection do not appear to be the sole cause of thymic atrophy. $\mathrm{T}$ cell differentiation only occurs in the thymic microenvironment, which is supported by TECs. By performing thymic transplants, we show that the stroma of $M$. avium-atrophied thymi are impaired in their capacity to support the differentiation of new $\mathrm{T}$ cells when provided with $\mathrm{BM}$ precursors from uninfected WT mice, which are able to give rise to $\mathrm{T}$ cells in thymic stroma of RAGKO mice. In a model of thymic atrophy during pregnancy, thymic stromal cells were shown to have limited capacity to produce chemokines essential for T cell precursors homing to the thymus, including CCL25, CXCL12, CCL21 and CCL19 (59). The limited ability to recruit $\mathrm{BM} \mathrm{T}$ cell precursors to the thymus could be a mechanism consistent with our previous observation that a reduction on the most immature thymocytes (ETPs; T cell precursor cells that just entered the thymus) occurs after infection with $M$. avium strain 25291 (10). However, we show that the expression in the thymus of Ccl25 and Cxcl12 is increased after infection with M. avium strain 25291. P-selectin, that is essential for the rolling and entry of $\mathrm{T}$ cell precursors in the thymus, is also increased in thymi from infected WT mice. These data led us to propose that there are limitations in the thymic stroma to retain T cell precursors and/ or support $\mathrm{T}$ cell differentiation itself. Long-term infection with the high virulence strain 25291 leads to alteration in the proportion of the two main thymic regions, namely a reduction in the cortex and a proportional increase in the medulla, that might simply be associated with lack of thymocytes. In some mice, but not all, thymi from 70 dpi reveal a lack of clear distinction between cortex and medulla and an overall increased expression of K5 and K8. This type of disorganized thymic structure has been previously observed during HIV (60-62), MuLV (63), Leishmania infantum (64) and $P$. berghei (65) infections.

It is possible that infection with $M$. avium strain 25291 impairs thymocyte differentiation itself, either by blocking a certain differentiation stage or by inducing thymocyte cell death. We observed increased expression of Il6 in the thymus of infected mice, which is in accordance with a study showing that over-expression of IL-6 inhibits the differentiation of double negative (DN) thymocytes in a model of $T$. cruzi infectioninduced thymic atrophy. Increased GC, IFN $\gamma, \mathrm{TNF}$ and NO levels have been associated with augmented thymocyte apoptosis during infection and other conditions (25-28, 66-68). We found a reduction in the percentage of viable (Annexin $\mathrm{V}^{-} \mathrm{PI}^{-}$) thymocytes after infection, independent of IFN $\gamma$ and iNOS. During $\mathrm{T}$ cell differentiation, all the non-positively selected thymocytes die by programmed cell death, which represents around $90 \%$ of the thymocytes. For this reason, the thymus is a specialized organ for dead cell clearance (69). This makes it challenging to study cell death in the thymus since dead cells are difficult to be tracked. This rapid clearance could explain why only a slight decrease in the percentage of viable thymocytes is observed after infection. Still, an increase in thymocyte death is consistent with most infection-induced thymic atrophy models, in which increased thymocyte apoptosis is suggested to be the main mechanism $(19,70-77)$. We detect an increase in Tnf mRNA expression in the thymus after infection with $M$. avium strain 25291, which was associated with thymocyte apoptosis in other models $(26,66,67)$. However, the increase in thymocyte apoptosis was also observed in WT mice infected with M. avium strain 2447, and in IFN $\gamma \mathrm{KO}$ and iNOSKO mice infected with strain 25291, all conditions without infection-induced thymic atrophy (10). This lack of correlation implies that while cell apoptosis may contribute to $M$. avium-induced thymic atrophy, it is not the sole mechanism. Additionally, we observed a decrease in the percentage of thymocytes positive for active 
caspase-3. Thus, while thymocytes are dying by apoptosis, apoptosis may be mediated by a mechanism other than activation of caspase-3. However, as caspase-3 is fundamental for $\mathrm{T}$ cell differentiation in the thymus (78), the reduction in the percentage of cells expressing the active form of this enzyme suggests that infection is affecting other unexplored parameters of thymocyte differentiation, in an iNOS dependent manner. Finally, WT infected mice have an increase in the percentage of necrotic/late apoptotic thymocytes, which is not observed in IFN $\gamma \mathrm{KO}$ and iNOSKO infected mice. As this increase is only detected at late time points (70 dpi), and thymic atrophy is already evident earlier (10), this implies that the appearance of necrotic/late apoptotic thymocytes is not the driver of M. aviuminduced thymic atrophy.

In conclusion, we show that the mechanism of M. aviuminduced premature thymic atrophy results from the association of several factors that seems to be cumulative: (1) alterations of the T cell precursors in the BM; (2) impaired ability of BM precursors to commit to thymocyte differentiation once within the thymus; (3) reduced capacity of thymic stromal cells to sustain $\mathrm{T}$ cell differentiation; and (4) IFN $\gamma$ - and iNOS-independent thymocyte apoptosis. Together, our data shows that infection with $M$. avium strain 25291 induces IFN $\gamma$ production that alters BM T cell precursors but that other alterations, independent of IFN $\gamma$ and iNOS, are also required. This is probably associated with the parallel production of IL-6, TNF and/or other pro-inflammatory cytokines. $\mathrm{T}$ cell precursors that reach the thymus encounter a harsh microenvironment affected by prolonged inflammation, which impairs their ability to differentiate, and/or leads to death, and culminates in premature thymic atrophy.

\section{DATA AVAILABILITY STATEMENT}

The raw data supporting the conclusions of this article will be made available by the authors, without undue reservation.

\section{ETHICS STATEMENT}

The animal study was reviewed and approved by in accordance with the recommendations of the European Convention for the Protection of Vertebrate Animals Used for Experimental and Other Scientific Purposes (ETS 123) and 86/609/EEC Directive and Portuguese rules (DL 129/92). The animal facility and people directly involved in animal experiments were certified by the

\section{REFERENCES}

1. Aspinall R. Age-Related Changes in the Function of T Cells. Microsc Res Tech (2003) 62:508-13. doi: 10.1002/jemt.10412

2. Francis IR, Glazer GM, Bookstein FL, Gross BH. The Thymus: Reexamination of Age-Related Changes in Size and Shape. AJR Am J Roentgenol (1985) 145:249-54. doi: 10.2214/ajr.145.2.249

3. Douek DC, McFarland RD, Keiser PH, Gage EA, Massey JM, Haynes BF, et al. Changes in Thymic Function With Age and During the Treatment of HIV Infection. Nature (1998) 396:690-5. doi: 10.1038/25374
Portuguese regulatory entity - Direção Geral de Alimentação e Veterinária (DGAV); the animal experimental protocols were approved by DGAV (\# 015584), or by the Institutional Animal Care and Use Committee at the University of Massachusetts Medical School (Animal Welfare A3306-01), using the recommendations from the Guide for the Care and Use of Laboratory Animals of the National Institutes of Health and the Office of Laboratory Animal Welfare.

\section{AUTHOR CONTRIBUTIONS}

PB-S, MC-N, and RA conceptualized, designed, and supervised the study. PB-S performed the experiments and processed the samples, analyzed the data, prepared the figures, performed the statistical analysis, and drafted the first version of the manuscript. RM-M, CN, SR, CS-M, MB, GA, and DS collected and processed samples. MC-N, $\mathrm{RA}$, and SB assembled the funding for experiments. All authors contributed to the article and approved the submitted version.

\section{FUNDING}

This work has been funded by National funds, through the Foundation for Science and Technology (FCT) - project UIDB/ $50026 / 2020$ and UIDP/50026/2020; by ICVS Scientific Microscopy Platform, member of the national infrastructure PPBI - Portuguese Platform of Bioimaging (PPBI-POCI-010145-FEDER-022122); and by a doctoral fellowship to PB-S (SFRH/BD/73544/2010). Experiments performed at University of Massachusetts Medical School were funded through a grant from the National Institutes of Health, R01 AI106725.

\section{ACKNOWLEDGMENTS}

We would like to acknowledge the preprint version of this manuscript on bioRxiv 2021.02.23.432464; https://doi.org/10. $1101 / 2021.02 .23 .432464$.

\section{SUPPLEMENTARY MATERIAL}

The Supplementary Material for this article can be found online at: https://www.frontiersin.org/articles/10.3389/fimmu.2021.696415/ full\#supplementary-material

4. Sauce D, Larsen M, Fastenackels S, Duperrier A, Keller M, GrubeckLoebenstein B, et al. Evidence of Premature Immune Aging in Patients Thymectomized During Early Childhood. J Clin Invest (2009) 119:3070-8. doi: $10.1172 /$ JCI39269

5. Heitger A, Neu N, Kern H, Panzer-Grumayer ER, Greinix H, Nachbaur D, et al. Essential Role of the Thymus to Reconstitute Naive (CD45RA+) THelper Cells After Human Allogeneic Bone Marrow Transplantation. Blood (1997) 90:850-7. doi: 10.1182/blood.V90.2.850.850_850_857

6. Smith KY, Valdez H, Landay A, Spritzler J, Kessler HA, Connick E, et al. Thymic Size and Lymphocyte Restoration in Patients With Human 
Immunodeficiency Virus Infection After 48 Weeks of Zidovudine, Lamivudine, and Ritonavir Therapy. J Infect Dis (2000) 181:141-7. doi: $10.1086 / 315169$

7. Nunes-Alves C, Nobrega C, Behar SM, Correia-Neves M. Tolerance has its Limits: How the Thymus Copes With Infection. Trends Immunol (2013) 34:502-10. doi: 10.1016/j.it.2013.06.004

8. Luo M, Xu L, Qian Z, Sun X. Infection-Associated Thymic Atrophy. Front Immunol (2021) 12:652538. doi: 10.3389/fimmu.2021.652538

9. Nobrega C, Cardona PJ, Roque S, Pinto do OP, Appelberg R, Correia-Neves M. The Thymus as a Target for Mycobacterial Infections. Microbes Infect (2007) 9:1521-9. doi: 10.1016/j.micinf.2007.08.006

10. Borges M, Barreira-Silva P, Flórido M, Jordan MB, Correia-Neves M, Appelberg R. Molecular and Cellular Mechanisms of Mycobacterium Avium-Induced Thymic Atrophy. J Immunol (2012) 189(7):3600-8. doi: 10.4049/jimmunol.1201525

11. Florido M, Goncalves AS, Silva RA, Ehlers S, Cooper AM, Appelberg R. Resistance of Virulent Mycobacterium Avium to Gamma InterferonMediated Antimicrobial Activity Suggests Additional Signals for Induction of Mycobacteriostasis. Infect Immun (1999) 67:3610-8. doi: 10.1128/ IAI.67.7.3610-3618.1999

12. de Meis J, Aurelio Farias-de-Oliveira D, Nunes Panzenhagen PH, Maran N, Villa-Verde DM, Morrot A, et al. Thymus Atrophy and Double-Positive Escape are Common Features in Infectious Diseases. J Parasitol Res (2012) 2012:574020. doi: 10.1155/2012/574020

13. Gameiro J, Nagib PR, Andrade CF, Villa-Verde DM, Silva-Barbosa SD, Savino W, et al. Changes in Cell Migration-Related Molecules Expressed by Thymic Microenvironment During Experimental Plasmodium Berghei Infection: Consequences on Thymocyte Development. Immunology (2010) 129:24856. doi: 10.1111/j.1365-2567.2009.03177.x

14. Savino W, Dardenne M, Velloso LA, Dayse Silva-Barbosa S. The Thymus is a Common Target in Malnutrition and Infection. Br J Nutr (2007) 98 Suppl 1: S11-6. doi: 10.1017/S0007114507832880

15. Leite de Moraes MD, Minoprio P, Dy M, Dardenne M, Savino W, HontebeyrieJoskowicz M. Endogenous IL-10 and IFN-Gamma Production Controls Thymic Cell Proliferation in Mice Acutely Infected by Trypanosoma Cruzi. Scand J Immunol (1994) 39:51-8. doi: 10.1111/j.1365-3083.1994.tb03339.x

16. Deobagkar-Lele M, Chacko SK, Victor ES, Kadthur JC, Nandi D. InterferonGamma- and Glucocorticoid-Mediated Pathways Synergize to Enhance Death of CD4(+) CD8(+) Thymocytes During Salmonella Enterica Serovar Typhimurium Infection. Immunology (2013) 138:307-21. doi: 10.1111/ imm. 12047

17. Farias-de-Oliveira DA, Villa-Verde DM, Nunes Panzenhagen PH, Silva dos Santos D, Berbert LR, Savino W, et al. Caspase- 8 and Caspase-9 Mediate Thymocyte Apoptosis in Trypanosoma Cruzi Acutely Infected Mice. J Leukoc Biol (2013) 93:227-34. doi: 10.1189/jlb.1211589

18. Morrot A, Barreto de Albuquerque J, Berbert LR, de Carvalho Pinto CE, de Meis J, Savino W. Dynamics of Lymphocyte Populations During Trypanosoma Cruzi Infection: From Thymocyte Depletion to Differential Cell Expansion/Contraction in Peripheral Lymphoid Organs. J Trop Med (2012) 2012:747185. doi: 10.1155/2012/747185

19. Souto PC, Brito VN, Gameiro J, da Cruz-Hofling MA, Verinaud L. Programmed Cell Death in Thymus During Experimental Paracoccidioidomycosis. Med Microbiol Immunol (2003) 192:225-9. doi: $10.1007 / \mathrm{s} 00430-003-0180-3$

20. Lepletier A, de Almeida L, Santos L, da Silva Sampaio L, Paredes B, Gonzalez FB, et al. Early Double-Negative Thymocyte Export in Trypanosoma Cruzi Infection is Restricted by Sphingosine Receptors and Associated With Human Chagas Disease. PloS Negl Trop Dis (2014) 8:e3203. doi: 10.1371/ journal.pntd.0003203

21. Morrot A, Terra-Granado E, Perez AR, Silva-Barbosa SD, Milicevic NM, Farias-de-Oliveira DA, et al. Chagasic Thymic Atrophy Does Not Affect Negative Selection But Results in the Export of Activated CD4+CD8+ T Cells in Severe Forms of Human Disease. PloS Negl Trop Dis (2011) 5:e1268. doi: 10.1371/journal.pntd.0001268

22. Perez AR, Berbert LR, Lepletier A, Revelli S, Bottasso O, Silva-Barbosa SD, et al. TNF-Alpha is Involved in the Abnormal Thymocyte Migration During Experimental Trypanosoma Cruzi Infection and Favors the Export of Immature Cells. PloS One (2012) 7:e34360. doi: 10.1371/journal.pone.0034360
23. Cardenas Palomo LF, de Souza Matos DC, Chaves Leal E, Bertho AL, Marcovistz R. Lymphocyte Subsets and Cell Proliferation Analysis in Rabies-Infected Mice. J Clin Lab Immunol (1995) 46:49-61.

24. Dooley J, Liston A. Molecular Control Over Thymic Involution: From Cytokines and microRNA to Aging and Adipose Tissue. Eur J Immunol (2012) 42:1073-9. doi: 10.1002/eji.201142305

25. Cohen O, Kfir-Erenfeld S, Spokoini R, Zilberman Y, Yefenof E, Sionov RV. Nitric Oxide Cooperates With Glucocorticoids in Thymic Epithelial CellMediated Apoptosis of Double Positive Thymocytes. Int Immunol (2009) 21:1113-23. doi: 10.1093/intimm/dxp079

26. Fayad R, Sennello JA, Kim SH, Pini M, Dinarello CA, Fantuzzi G. Induction of Thymocyte Apoptosis by Systemic Administration of Concanavalin A in Mice: Role of TNF-Alpha, IFN-Gamma and Glucocorticoids. Eur J Immunol (2005) 35:2304-12. doi: 10.1002/eji.200526062

27. Gordon SA, Abou-Jaoude W, Hoffman RA, McCarthy SA, Kim YM, Zhou X, et al. Nitric Oxide Induces Murine Thymocyte Apoptosis by Oxidative Injury and a P53-Dependent Mechanism. J Leukoc Biol (2001) 70:87-95. doi: $10.1189 / \mathrm{jlb} .70 .1 .87$

28. Tai XG, Toyo-oka K, Yamamoto N, Yashiro Y, Mu J, Hamaoka T, et al. Expression of an Inducible Type of Nitric Oxide (NO) Synthase in the Thymus and Involvement of NO in Deletion of TCR-Stimulated DoublePositive Thymocytes. J Immunol (1997) 158:4696-703.

29. Choi HH, Kim KK, Kim KD, Kim HJ, Jo EK, Song CH. Effects of Mycobacterial Infection on Proliferation of Hematopoietic Precursor Cells. Microbes Infect (2011) 13:1252-60. doi: 10.1016/j.micinf.2011.08.001

30. Baldridge MT, King KY, Boles NC, Weksberg DC, Goodell MA. Quiescent Haematopoietic Stem Cells are Activated by IFN-Gamma in Response to Chronic Infection. Nature (2010) 465:793-7. doi: 10.1038/nature09135

31. Matsumura T, Ato M, Ikebe T, Ohnishi M, Watanabe H, Kobayashi K. Interferon-Gamma-Producing Immature Myeloid Cells Confer Protection Against Severe Invasive Group A Streptococcus Infections. Nat Commun (2012) 3:678. doi: 10.1038/ncomms1677

32. Burberry A, Zeng MY, Ding L, Wicks I, Inohara N, Morrison SJ, et al. Infection Mobilizes Hematopoietic Stem Cells Through Cooperative NODLike Receptor and Toll-Like Receptor Signaling. Cell Host Microbe (2014) 15:779-91. doi: 10.1016/j.chom.2014.05.004

33. Moses AV, Williams S, Heneveld ML, Strussenberg J, Rarick M, Loveless M, et al. Human Immunodeficiency Virus Infection of Bone Marrow Endothelium Reduces Induction of Stromal Hematopoietic Growth Factors. Blood (1996) 87:919-25. doi: 10.1182/blood.V87.3.919.bloodjournal873919

34. Maltby S, Hansbro NG, Tay HL, Stewart J, Plank M, Donges B, et al. Production and Differentiation of Myeloid Cells Driven by Proinflammatory Cytokines in Response to Acute Pneumovirus Infection in Mice. J Immunol (2014) 193:4072-82. doi: 10.4049/jimmunol.1400669

35. Singh P, Yao Y, Weliver A, Broxmeyer HE, Hong SC, Chang CH. Vaccinia Virus Infection Modulates the Hematopoietic Cell Compartments in the Bone Marrow. Stem Cells (2008) 26:1009-16. doi: 10.1634/stemcells.2007-0461

36. Belyaev NN, Brown DE, Diaz AI, Rae A, Jarra W, Thompson J, et al. Induction of an IL7-R(+)c-Kit(hi) Myelolymphoid Progenitor Critically Dependent on IFN-Gamma Signaling During Acute Malaria. Nat Immunol (2010) 11:47785. doi: $10.1038 /$ ni. 1869

37. Frankenburg S, Londner MV, Greenblatt CL. Cellular Changes in the Bone Marrow of Plasmodium Berghei-Infected Mice. II. Blast Transformation and Phagocytosis. Cell Immunol (1980) 55:185-90. doi: 10.1016/0008-8749(80)90149-5

38. Mabbott N, Sternberg J. Bone Marrow Nitric Oxide Production and Development of Anemia in Trypanosoma Brucei-Infected Mice. Infect Immun (1995) 63:1563-6. doi: 10.1128/iai.63.4.1563-1566.1995

39. Zhao X, Ren G, Liang L, Ai PZ, Zheng B, Tischfield JA, et al. Brief Report: Interferon-Gamma Induces Expansion of Lin(-)Sca-1(+)C-Kit(+) Cells. Stem Cells (2010) 28:122-6. doi: 10.1002/stem.252

40. Smith JNP, Zhang Y, Li JJ, McCabe A, Jo HJ, Maloney J, et al. Type I IFNs Drive Hematopoietic Stem and Progenitor Cell Collapse via Impaired Proliferation and Increased RIPK1-Dependent Cell Death During ShockLike Ehrlichial Infection. PloS Pathog (2018) 14(8):e1007234. doi: 10.1371/ journal.ppat.1007234

41. Kong Y, Li Y, Zhang W, Yuan S, Winkler R, Krohnert U, et al. Sepsis-Induced Thymic Atrophy Is Associated With Defects in Early Lymphopoiesis. Stem Cells (2016) 34(12):2902-15. doi: 10.1002/stem.2464 
42. Carbajosa S, Gea S, Chillón-Marinas C, Poveda C, Maza M del C, Fresno M, et al. Altered Bone Marrow Lymphopoiesis and Interleukin-6-Dependent Inhibition of Thymocyte Differentiation Contribute to Thymic Atrophy During Trypanosoma Cruzi Infection. Oncotarget (2017) 8:17551-61. doi: 10.18632/oncotarget.14886

43. Lykens JE, Terrell CE, Zoller EE, Divanovic S, Trompette A, Karp CL, et al. Mice With a Selective Impairment of IFN-Gamma Signaling in Macrophage Lineage Cells Demonstrate the Critical Role of IFN-Gamma-Activated Macrophages for the Control of Protozoan Parasitic Infections In Vivo. J Immunol (2010) 184:877-85. doi: 10.4049/jimmunol.0902346

44. MacMicking JD, Nathan C, Hom G, Chartrain N, Fletcher DS, Trumbauer M, et al. Altered Responses to Bacterial Infection and Endotoxic Shock in Mice Lacking Inducible Nitric Oxide Synthase. Cell (1995) 81:641-50. doi: 10.1016/ 0092-8674(95)90085-3

45. Florido M, Pearl JE, Solache A, Borges M, Haynes L, Cooper AM, et al. Gamma Interferon-Induced T-Cell Loss in Virulent Mycobacterium Avium Infection. Infect Immun (2005) 73:3577-86. doi: 10.1128/IAI.73.6.3577-3586.2005

46. Gomes AC, Moreira AC, Silva T, Neves JV, Mesquita G, Almeida AA, et al. IFN- $\gamma$-Dependent Reduction of Erythrocyte Life Span Leads to Anemia During Mycobacterial Infection. J Immunol (2019) 203(9):2485-96. doi: 10.4049/jimmunol.1900382

47. Spangrude GJ, Heimfeld S, Weissman IL. Purification and Characterization of Mouse Hematopoietic Stem Cells. Science (80-) (1988) 241:58-62. doi: $10.1126 /$ science. 2898810

48. Iwasaki H, Akashi K. Hematopoietic Developmental Pathways: On Cellular Basis. Oncogene (2007) 26:6687-96. doi: 10.1038/sj.onc.1210754

49. de Bruin AM, Demirel O, Hooibrink B, Brandts CH, Nolte MA. InterferonGamma Impairs Proliferation of Hematopoietic Stem Cells in Mice. Blood (2013) 121:3578-85. doi: 10.1182/blood-2012-05-432906

50. Essers MA, Offner S, Blanco-Bose WE, Waibler Z, Kalinke U, Duchosal MA, et al. IFNalpha Activates Dormant Haematopoietic Stem Cells In Vivo. Nature (2009) 458:904-8. doi: 10.1038/nature07815

51. MacNamara KC, Oduro K, Martin O, Jones DD, McLaughlin M, Choi K, et al. Infection-Induced Myelopoiesis During Intracellular Bacterial Infection is Critically Dependent Upon IFN-Gamma Signaling. J Immunol (2011) 186:1032-43. doi: 10.4049/jimmunol.1001893

52. McCabe A, Zhang $\mathrm{Y}$, Thai V, Jones M, Jordan MB, MacNamara KC. Macrophage-Lineage Cells Negatively Regulate the Hematopoietic Stem Cell Pool in Response to Interferon Gamma at Steady State and During Infection. Stem Cells (2015) 33:2294-305. doi: 10.1002/stem.2040

53. Pietras EM, Lakshminarasimhan R, Techner JM, Fong S, Flach J, Binnewies M, et al. Re-Entry Into Quiescence Protects Hematopoietic Stem Cells From the Killing Effect of Chronic Exposure to Type I Interferons. J Exp Med (2014) 211:245-62. doi: 10.1084/jem.20131043

54. Pronk CJ, Veiby OP, Bryder D, Jacobsen SE. Tumor Necrosis Factor Restricts Hematopoietic Stem Cell Activity in Mice: Involvement of Two Distinct Receptors. J Exp Med (2011) 208:1563-70. doi: 10.1084/jem.20110752

55. Kaur S, Raggatt LJ, Batoon L, Hume DA, Levesque JP, Pettit AR. Role of Bone Marrow Macrophages in Controlling Homeostasis and Repair in Bone and Bone Marrow Niches. Semin Cell Dev Biol (2017) 61:12-21. doi: 10.1016/ j.semcdb.2016.08.009

56. Winkler IG, Sims NA, Pettit AR, Barbier V, Nowlan B, Helwani F, et al. Bone Marrow Macrophages Maintain Hematopoietic Stem Cell (HSC) Niches and Their Depletion Mobilizes HSCs. Blood (2010) 116(23):4815-28. doi: 10.1182/ blood-2009-11-253534

57. Klei TRL, Meinderts SM, van den Berg TK, van Bruggen R. From the Cradle to the Grave: The Role of Macrophages in Erythropoiesis and Erythrophagocytosis. Front Immunol (2017) 8:73. doi: 10.3389/fimmu.2017.00073

58. Raykova VD, Glibetic M, Ofenstein JP, Aranda JV. Nitric Oxide-Dependent Regulation of Pro-Inflammatory Cytokines in Group B Streptococcal Inflammation of Rat Lung. Ann Clin Lab Sci (2003) 33(1):62-7.

59. Laan M, Haljasorg U, Kisand K, Salumets A, Peterson P. Pregnancy-Induced Thymic Involution is Associated With Suppression of Chemokines Essential for T-Lymphoid Progenitor Homing. Eur J Immunol (2016) 46:2008-17. doi: 10.1002/eji.201646309

60. Douek DC, Betts MR, Hill BJ, Little SJ, Lempicki R, Metcalf JA, et al. Evidence for Increased $\mathrm{T}$ Cell Turnover and Decreased Thymic Output in HIV Infection. J Immunol (2001) 167:6663-8. doi: 10.4049/jimmunol.167.11.6663
61. Schmitt N, Nugeyre MT, Scott-Algara D, Cumont MC, Barre-Sinoussi F, Pancino G, et al. Differential Susceptibility of Human Thymic Dendritic Cell Subsets to X4 and R5 HIV-1 Infection. AIDS (2006) 20:533-42. doi: 10.1097/ 01.aids.0000210607.63138.bc

62. Stanley SK, McCune JM, Kaneshima H, Justement JS, Sullivan M, Boone E, et al. Human Immunodeficiency Virus Infection of the Human Thymus and Disruption of the Thymic Microenvironment in the SCID-Hu Mouse. J Exp Med (1993) 178:1151-63. doi: 10.1084/jem.178.4.1151

63. de Leval L, Deprez M, Colombi S, Humblet C, Defresne M-P, Boniver J, et al. Morphological Changes of Thymus in Retrovirus-Induced Murine Acquired Immunodeficiency Syndrome (MAIDS). Pathol - Res Pract (1995) 191:50612. doi: 10.1016/S0344-0338(11)80869-1

64. Losada-Barragán M, Umaña-Pérez A, Durães J, Cuervo-Escobar S, RodríguezVega A, Ribeiro-Gomes FL, et al. Thymic Microenvironment Is Modified by Malnutrition and Leishmania Infantum Infection. Front Cell Infect Microbiol (2019) 9:252. doi: 10.3389/fcimb.2019.00252

65. Andrade CF, Gameiro J, Nagib PRA, Carvalho BO, Talaisys RL, Costa FTM, et al. Thymic Alterations in Plasmodium Berghei-Infected Mice. Cell Immunol (2008) 253:1-4. doi: 10.1016/j.cellimm.2008.06.001

66. Belhacene N, Gamas P, Goncalves D, Jacquin M, Beneteau M, Jacquel A, et al. Severe Thymic Atrophy in a Mouse Model of Skin Inflammation Accounts for Impaired TNFR1 Signaling. PloS One (2012) 7:e47321. doi: 10.1371/ journal.pone.0047321

67. Hernandez-Caselles T, Stutman O. Immune Functions of Tumor Necrosis Factor. I. Tumor Necrosis Factor Induces Apoptosis of Mouse Thymocytes and can Also Stimulate or Inhibit IL-6-Induced Proliferation Depending on the Concentration of Mitogenic Costimulation. J Immunol (1993) 151:3999-4012.

68. Wyllie AH. Glucocorticoid-Induced Thymocyte Apoptosis is Associated With Endogenous Endonuclease Activation. Nature (1980) 284:555-6. doi: 10.1038/ $284555 \mathrm{a} 0$

69. Krueger A, Ziętara N. Łyszkiewicz M. T Cell Development by the Numbers. Trends Immunol (2017) 38(2):128-39. doi: 10.1016/j.it.2016.10.007

70. Brito VN, Souto PC, Cruz-Hofling MA, Ricci LC, Verinaud L. Thymus Invasion and Atrophy Induced by Paracoccidioides Brasiliensis in BALB/C Mice. Med Mycol (2003) 41:83-7. doi: 10.1080/714043902

71. Chen W, Kuolee R, Austin JW, Shen H, Che Y, Conlan JW. Low Dose Aerosol Infection of Mice With Virulent Type A Francisella Tularensis Induces Severe Thymus Atrophy and CD4+CD8+ Thymocyte Depletion. Microb Pathog (2005) 39:189-96. doi: 10.1016/j.micpath.2005.08.005

72. Leite-de-Moraes MC, Hontebeyrie-Joskowicz M, Dardenne M, Savino W. Modulation of Thymocyte Subsets During Acute and Chronic Phases of Experimental Trypanosoma Cruzi Infection. Immunology (1992) 77:95-8.

73. Marcovistz R, Bertho AL, Matos DC. Relationship Between Apoptosis and Thymocyte Depletion in Rabies-Infected Mice. Braz J Med Biol Res (1994) 27:1599-603.

74. Savino W, Dardenne M, Marche C, Trophilme D, Dupuy JM, Pekovic D, et al. Thymic Epithelium in AIDS. An Immunohistologic Study. Am J Pathol (1986) 122:302-7.

75. Savino W, Leite-de-Moraes MC, Hontebeyrie-Joskowicz M, Dardenne M. Studies on the Thymus in Chagas' Disease. I. Changes in the Thymic Microenvironment in Mice Acutely Infected With Trypanosoma Cruzi. Eur J Immunol (1989) 19:1727-33. doi: 10.1002/eji.1830190930

76. Seixas E, Ostler D. Plasmodium Chabaudi Chabaudi (AS): Differential Cellular Responses to Infection in Resistant and Susceptible Mice. Exp Parasitol (2005) 110:394-405. doi: 10.1016/j.exppara.2005.03.024

77. Watson SR, Redington TJ, Miller TB, Bullock WE. Flow Microfluorometry Analysis of Alterations in T-Lymphocyte Subsets During Murine Listeriosis. Infect Immun (1984) 45:372-7. doi: 10.1128/iai.45.2.372-377.1984

78. Jiang D, Zheng L, Lenardo MJ. Caspases in T-Cell Receptor-Induced Thymocyte Apoptosis. Cell Death Differ (1999) 6:402-11. doi: 10.1038/ sj.cdd. 4400513

Conflict of Interest: The authors declare that the research was conducted in the absence of any commercial or financial relationships that could be construed as a potential conflict of interest.

Publisher's Note: All claims expressed in this article are solely those of the authors and do not necessarily represent those of their affiliated organizations, or those of 
the publisher, the editors and the reviewers. Any product that may be evaluated in this article, or claim that may be made by its manufacturer, is not guaranteed or endorsed by the publisher.

Copyright $\odot 2021$ Barreira-Silva, Melo-Miranda, Nobrega, Roque, Serre-Miranda, Borges, Armada, de Sá Calçada, Behar, Appelberg and Correia-Neves. This is an open-access article distributed under the terms of the Creative Commons Attribution License (CC BY). The use, distribution or reproduction in other forums is permitted, provided the original author(s) and the copyright owner(s) are credited and that the original publication in this journal is cited, in accordance with accepted academic practice. No use, distribution or reproduction is permitted which does not comply with these terms. 